\title{
Hypocoercivity of Linear Degenerately Dissipative Kinetic Equations
}

\author{
Renjun DuAn \\ Department of Mathematics, The Chinese University of Hong Kong \\ Shatin, Hong Kong \\ E-mail: rjduan@math.cuhk.edu.hk
}

\begin{abstract}
In this paper we develop a general approach of studying the hypocoercivity for a class of linear kinetic equations with both transport and degenerately dissipative terms. As concrete examples, the relaxation operator, Fokker-Planck operator and linearized Boltzmann operator are considered when the spatial domain takes the whole space or torus and when there is a confining force or not. The key part of the developed approach is to construct some equivalent temporal energy functionals for obtaining time rates of the solution trending towards equilibrium in some Hilbert spaces. The result in the case of the linear Boltzmann equation with confining forces is new. The proof mainly makes use of the macro-micro decomposition combined with Kawashima's argument on dissipation of the hyperbolic-parabolic system. At the end, a Korn-type inequality with probability measure is provided to deal with dissipation of momentum components.
\end{abstract}

Keywords: Hypocoercivity; semigroup; kinetic equation; dissipation

\section{Introduction}

1.1 Problems. In this paper, we consider the linear kinetic equation with both transport and dissipative terms in the form of

$$
\partial_{t} u+\mathrm{T} u=\mathrm{L} u
$$

Here, the unknown is $u=u(t, x, \xi)$ with $t>0, x \in \Omega=\mathbb{R}^{d}$ or $\mathbb{T}^{d}, \xi \in \mathbb{R}^{d} . d \geq 1$ denotes the space dimension. $\mathrm{T}=\xi \cdot \nabla_{x}-\nabla_{x} V \cdot \nabla_{\xi}$ is a transport operator. $V=V(x)$ is a given stationary external forcing. $\mathrm{L}: L_{\xi}^{2} \rightarrow L_{\xi}^{2}$ is a linear, local, self-adjoint and non-positive operator with ker $L \neq\{0\}$. Moreover, $L$ is degenerately dissipative in the sense that there is a constant $\lambda_{L}>0$ such that

$$
\int_{\mathbb{R}^{d}} u \mathrm{~L} u d \xi \leq-\lambda_{\mathrm{L}}\|\{\mathrm{I}-\mathrm{P}\} u\|_{\mathcal{H}_{\xi}}^{2}
$$

where $\mathcal{H}_{\xi} \subset L_{\xi}^{2}$ is a Hilbert space with norm $\|\cdot\|_{\mathcal{H}_{\xi}}, \mathrm{I}$ is an identity operator and $\mathrm{P}$ is an orthogonal velocity projection operator from $L_{\xi}^{2}$ to ker $\mathrm{L}$. Given initial data $u(0, x, \xi)=u_{0}(x, \xi)$, the solution of 1.1 formally takes the form of $u(t)=e^{t \mathrm{~B}} u_{0}$ with $\mathrm{B}=: \mathrm{L}-\mathrm{T}$. The goal of this paper is to study time-decay rates of $e^{t \mathrm{~B}} u_{0}$ in some Hilbert space under some conditions on $V$ and $u_{0}$ as time tends to infinity. 
1.2 Literature. The time rate of convergence of solutions to equilibrium is an important issue in the study of evolution equations. For the linear kinetic equation in the form of (1.1), the main difficulty lies in the fact that the linearized operator is degenerate in a nontrivial finite dimensional space since conservation laws exist for general physical models. However, the interaction between the transport part and the degenerate dissipative part can lead to convergence to equilibrium. This property is called hypocoercivity [28].

There have been several well-established methods to study the rate of convergence for the kinetic models such as the relaxation equation, Fokker-Planck equation, Boltzmann equation and Landau equation. Since the literature on this subject is so huge, we only mention some of them which are related to the study of this paper. The non-constructive method by spectral analysis to obtain the exponential rates for the Boltzmann equation with hard potentials on torus was firstly provided by Ukai 26]. The recent refinement of results of 26] can be found in [27. Energy method of the Boltzmann equation was developed by Liu-Yu [22], Liu-Yang-Yu 21] and Guo [14, 13. Energy method combined with additional techniques such as velocity weight estimates or spectral analysis [24, 25, 10] was also applied to obtain time decay rates in the framework of perturbations. Another powerful tool is entropy method which works in the non-perturbation framework. By using this method, Desvillettes-Villani 4 obtained firstly the almost exponential rate of convergence of solutions to the Boltzmann equation on torus with soft potentials for large initial data under the additional regularity conditions. Concerning the Fokker-Planck equation, see [1] and references therein. In addition, on the basis of the spectral analysis, the hypoelliptic theory of the Fokker-Planck equation or relaxation Boltzmann equation was developed by Hérau-Nier [16] and Hérau [15, 17.

Recently, some general theory on hypocoercivity was provided in [23, 5, 28. By constructing some proper Lyapunov functional defined over the Hilbert space, Mouhot-Neumann 23. obtained the exponential rates of convergence in $H^{1}$-norm for some kinetic models with general structures in the case of torus. An extension of [23] to models in the presence of a confining potential force was given by Dolbeault-Mouhot-Schmeiser [5], where $L^{2}$-norm was considered. Villani 28] also gave a systematic study of the Hypocoercivity theory. The result on the FokkerPlanck in this paper is inspired by [28]; we will give an alternative proof on the basis of a unified approach to be developed for different models here.

1.3 Notations. Define differential operators $\mathrm{X}_{i}, \mathrm{Y}_{i}(1 \leq i \leq d)$ by

$$
\mathrm{X}_{i} u=e^{-\frac{V(x)}{2}} \partial_{x_{i}}\left(e^{\frac{V(x)}{2}} u\right), \quad \mathbf{Y}_{i} u=e^{-\frac{|\xi|^{2}}{4}} \partial_{\xi_{i}}\left(e^{\frac{|\xi|^{2}}{4}} u\right) .
$$

Note that $\mathbf{X}_{i}, \mathbf{Y}_{i}$ are equivalent with $\mathbf{X}_{i}=\frac{1}{2} \partial_{x_{i}} V+\partial_{x_{i}}, \mathbf{Y}_{i} u=\frac{1}{2} \xi_{i}+\partial_{\xi_{i}}$. Introduce a norm $\|\cdot\|_{\mathcal{H}^{1}}$ by

$$
\|u\|_{\mathcal{H}^{1}}^{2}=\|u\|^{2}+\|\mathrm{X} u\|^{2}+\|\mathrm{Y} u\|^{2}
$$

for $u=u(x, \xi)$. Here and in the sequel, $\|\cdot\|$ means $L^{2}$-norm over $\mathbb{R}_{x}^{d} \times \mathbb{R}_{\xi}^{d}, \mathbf{X}=\left(\mathrm{X}_{1}, \mathrm{X}_{2}, \cdots, \mathrm{X}_{d}\right)$, $\mathrm{Y}=\left(\mathrm{Y}_{1}, \mathrm{Y}_{2}, \cdots, \mathrm{Y}_{d}\right)$, and $\|\mathrm{X} u\|^{2}=\sum_{i=1}^{d}\left\|\mathrm{X}_{i} u\right\|^{2},\|\mathrm{Y} u\|^{2}=\sum_{i=1}^{d}\left\|\mathrm{Y}_{i} u\right\|^{2}$. For simplicity, when a function under consideration is independent of velocity variable, $\|\cdot\|$ is also used to denote $L^{2}$-norm over $\mathbb{R}_{x}^{d}$ and $\partial_{i}=\partial_{x_{i}}$ without any confusion. For the inner products over $L_{x}^{2}$ and $L_{\xi}^{2}$, we use

$$
(g, h)=\int_{\Omega} f(x) g(x) d x,\langle g, h\rangle=\int_{\mathbb{R}^{d}} g(\xi) h(\xi) d \xi,
$$

respectively. For a function $w=w(\xi)$, define norms $|\cdot|_{w}$ and $\|\cdot\|_{w}$ by

$$
\begin{aligned}
|g|_{w}^{2} & =\int_{\mathbb{R}^{d}}\left|\nabla_{\xi} g(\xi)\right|^{2}+w(\xi)|g(\xi)|^{2} d \xi, \quad g=g(\xi), \\
\|g\|_{w}^{2} & =\iint_{\Omega \times \mathbb{R}^{d}}\left|\nabla_{\xi} g(x, \xi)\right|^{2}+w(\xi)|g(x, \xi)|^{2} d x d \xi, g=g(x, \xi) .
\end{aligned}
$$


For $q \geq 1$ and $\Omega=\mathbb{R}^{d}$, define

$$
Z_{q}=L_{\xi}^{2}\left(L_{x}^{q}\right)=L^{2}\left(\mathbb{R}_{\xi}^{d} ; L^{q}\left(\mathbb{R}_{x}^{d}\right)\right), \quad\|g\|_{Z_{q}}=\left(\int_{\mathbb{R}^{d}}\left(\int_{\mathbb{R}^{d}}|g(x, \xi)|^{q} d x\right)^{2 / q} d \xi\right)^{1 / 2}
$$

Denote two functions $M, \mathcal{M}$ by

$$
M(\xi)=(2 \pi)^{-d / 2} e^{-|\xi|^{2} / 2}, \quad \mathcal{M}(x, \xi)=e^{-V(x)} M(\xi),
$$

where $M$ is a normalized Maxwellian. $C$ denotes some positive (generally large) constant and $\lambda$ denotes some positive (generally small) constant, where both $C$ and $\lambda$ may take different values in different places. $A \sim B$ means $\lambda_{1} A \leq B \leq \lambda_{2} A$ for two generic constants $\lambda_{1}>0$ and $\lambda_{2}>0$. For an integrable function $g: \Omega \rightarrow \mathbb{R}$, its Fourier transform $\hat{g}$ is defined by

$$
\hat{g}(k)=\int_{\Omega} e^{-\mathrm{i} x \cdot k} g(x) d x, \quad x \cdot k=: \sum_{j=1}^{d} x_{j} k_{j}
$$

for $k \in \mathbb{R}^{d}$ if $\Omega=\mathbb{R}^{d}$ while $k \in \mathbb{Z}^{d}$ when $\Omega=\mathbb{T}^{d}$, where $\mathrm{i}=\sqrt{-1} \in \mathbb{C}$ is the imaginary unit. For two complex numbers $c_{1}, c_{2} \in \mathbb{C},\left(c_{1} \mid c_{2}\right)=c_{1} \cdot \bar{c}_{2}$ denotes the dot product over the complex field, where $\bar{c}_{2}$ is the complex conjugate of $c_{2}$.

1.4 Models. To the end, we shall consider three types of degenerately dissipative operators for $\mathrm{L}$ as follows.

Model 1. $\mathrm{L}$ is the linear relaxation operator

$$
\mathrm{L} u=-\left\{\mathrm{I}-\mathrm{P}_{0}\right\} u,
$$

where $\mathrm{P}_{0}: L_{\xi}^{2} \rightarrow \operatorname{span}\left\{M^{1 / 2}\right\}$ is an orthogonal velocity projection operator given by

$$
\mathrm{P}_{0} u=a M^{1 / 2}, \quad a=a^{u}=:\left\langle M^{1 / 2}, u\right\rangle .
$$

Notice that $\operatorname{Ker} \mathrm{L}=\operatorname{span}\left\{M^{1 / 2}\right\}$ and $-\mathrm{L}$ satisfies the identity

$$
-\int_{\mathbb{R}^{d}} u \mathrm{~L} u d \xi=\int_{\mathbb{R}^{d}}\left|\left\{\mathrm{I}-\mathrm{P}_{0}\right\} u\right|^{2} d \xi
$$

Moreover, if $f=\mathcal{M}+\mathcal{M}^{1 / 2} u$, then $f$ equivalently satisfies the original relaxation model:

$$
\partial_{t} f+\mathrm{\top} f=M \int_{\mathbb{R}^{d}} f d \xi-f
$$

Model 2. $\mathrm{L}$ is the linear self-adjoint Fokker-Planck operator

$$
\mathrm{L} u=\frac{1}{M^{1 / 2}} \nabla_{\xi} \cdot\left(M \nabla_{\xi}\left(\frac{u}{M^{1 / 2}}\right)\right) .
$$

It is well-known that $\operatorname{Ker} \mathrm{L}=\operatorname{span}\left\{M^{1 / 2}\right\}$ and $-\mathrm{L}$ satisfies the coercivity

$$
-\int_{\mathbb{R}^{d}} u \mathrm{~L} u d \xi \geq\left|\left\{\mathrm{I}-\mathrm{P}_{0}\right\} u\right|_{\nu}^{2}
$$


where $\nu=\nu(\xi)=1+|\xi|^{2}$ and $\mathbf{P}_{0}$ is still defined by 1.5). Instead of 1.7), it is more convenient to use another equivalent coercivity inequality as used in [8]. In fact, define $\mathrm{P}: L_{\xi}^{2} \rightarrow$ $\operatorname{span}\left\{M^{1 / 2}, \xi M^{1 / 2}\right\}$ as

$$
\left\{\begin{array}{l}
\mathrm{P} u=\mathrm{P}_{0} u \oplus \mathrm{P}_{1} u, \\
\mathrm{P}_{0} u=a M^{1 / 2}, \quad a=a^{u}=\left\langle M^{1 / 2}, u\right\rangle \\
\mathrm{P}_{1} u=b \cdot \xi M^{1 / 2}, \quad b=b^{u}=\left\langle\xi M^{1 / 2}, u\right\rangle .
\end{array}\right.
$$

Then, one can verify that

$$
-\int_{\mathbb{R}^{d}} u \mathrm{~L} u d \xi \geq \lambda|\{\mathrm{I}-\mathrm{P}\} u|_{\nu}^{2}+|b|^{2} .
$$

Similarly as before, if let $f=\mathcal{M}+\mathcal{M}^{1 / 2} u$ then $f$ equivalently satisfies the linear Fokker-Planck equation:

$$
\partial_{t} f+\mathrm{T} f=\nabla_{\xi} \cdot\left(\nabla_{\xi} f+\xi f\right) .
$$

Model 3. $\mathrm{L}$ is the linearized Boltzmann operator

$$
\mathrm{L} u=\frac{1}{M^{1 / 2}}\left[\mathrm{Q}\left(M, M^{1 / 2} u\right)+\mathrm{Q}\left(M^{1 / 2} u, M\right)\right],
$$

where $Q$ is the so-called bilinear collision operator defined by

$$
\begin{array}{r}
\mathrm{Q}(f, g)=\iint_{\mathbb{R}^{d} \times S^{d-1}}\left|\left(\xi-\xi_{*}\right) \cdot \omega\right|\left(f\left(\xi^{\prime}\right) g\left(\xi_{*}^{\prime}\right)-f(\xi) g\left(\xi_{*}\right)\right) d \omega d \xi_{*}, \\
\quad\left\{\begin{array}{l}
\xi^{\prime}=\xi-\left[\left(\xi-\xi_{*}\right) \cdot \omega\right] \omega \\
\xi_{*}^{\prime}=\xi_{*}+\left[\left(\xi-\xi_{*}\right) \cdot \omega\right] \omega
\end{array} \quad \omega \in S^{d-1}\right.
\end{array}
$$

for $f=f(\xi)$ and $g=g(\xi)$. Here, although the hard-sphere collision kernel in Q is supposed, all results of this paper still hold in the case of hard potentials and Maxwell molecules. For L, it is also well-known [2] that $\operatorname{dim} \operatorname{ker} \mathrm{L}=d+2$,

$$
\operatorname{ker} \mathrm{L}=\operatorname{span}\left\{M^{1 / 2}, \xi_{1} M^{1 / 2}, \cdots, \xi_{d} M^{1 / 2},|\xi|^{2} M^{1 / 2}\right\},
$$

and $-\mathrm{L}$ satisfies the coercivity

$$
-\int_{\mathbb{R}^{d}} u \mathrm{~L} u d \xi \geq \lambda \int_{\mathbb{R}^{d}} \nu(\xi)|\{\mathrm{I}-\mathrm{P}\} u|^{2} d \xi
$$

where we still used $\nu(\xi)$ to denote the collision frequency defined by

$$
\nu(\xi)=\iint_{\mathbb{R}^{d} \times S^{d-1}}\left|\left(\xi-\xi_{*}\right) \cdot \omega\right| M d \omega d \xi_{*},
$$

and also used $\mathrm{P}: L_{\xi}^{2} \rightarrow \operatorname{ker} \mathrm{L}$ to denote the orthogonal velocity projection operator. For convenience, corresponding to the $d+2$ dimensional space $[1.9], \mathrm{P}$ is written as

$$
\left\{\begin{array}{l}
\mathrm{P} u=\left\{a+b \cdot \xi+c\left(|\xi|^{2}-d\right)\right\} M^{1 / 2}, \\
a=a^{u}=\left\langle M^{1 / 2}, u\right\rangle, \\
b=b^{u}=\left\langle\xi M^{1 / 2}, u\right\rangle, \\
c=c^{u}=\frac{1}{2 d}\left\langle\left(|\xi|^{2}-d\right) M^{1 / 2}, u\right\rangle .
\end{array}\right.
$$


Therefore, $a, b, c$ mean mass, momentum and temperature components of macroscopic part Pu. If let $f=\mathcal{M}+\mathcal{M}^{1 / 2} u$ then $f$ satisfies the linear Boltzmann equation:

$$
\partial_{t} f+\mathrm{T} f=\mathrm{Q}(M, f)+\mathrm{Q}(f, M) .
$$

1.5 Main results. Let us state them in two cases which will be proved in terms of different analytical tools.

Theorem 1.1 (case of no force). Consider 1.1 where $\Omega=\mathbb{R}^{d}$ or $\mathbb{T}^{d}, d \geq 1, V=0$, and $\mathrm{L}$ is one of the linear relaxation operator, linear Fokker-Planck operator and linearized Boltzmann operator as defined in Model 1, Model 2 and Model 3, respectively. Let $e^{t \mathrm{~B}} u_{0}$ denote the corresponding solution for initial data $u_{0}=u_{0}(x, \xi)$.

Case $\left(\Omega=\mathbb{R}^{d}\right)$. Let $h=h(t, x, \xi)$ satisfy

$$
h(t, x, \xi) \perp \operatorname{ker} \mathrm{L}, \quad \forall t \geq 0, x \in \mathbb{R}^{d} .
$$

Let $1 \leq q \leq 2$. Then, for any $\alpha, \alpha^{\prime}$ with $\alpha^{\prime} \leq \alpha$ and $m=\left|\alpha-\alpha^{\prime}\right|$, there is a constant $C$ such that

$$
\left\|\partial_{x}^{\alpha} e^{t \mathrm{~B}} u_{0}\right\| \leq C(1+t)^{-\sigma_{q, m}}\left(\left\|\partial_{x}^{\alpha^{\prime}} u_{0}\right\|_{Z_{q}}+\left\|\partial_{x}^{\alpha} u_{0}\right\|\right)
$$

and

$$
\begin{gathered}
\left\|\partial_{x}^{\alpha} \int_{0}^{t} e^{-(t-s) \mathrm{B}} h(s) d s\right\|^{2} \\
\leq C \int_{0}^{t}(1+t-s)^{-2 \sigma_{q, m}}\left(\left\|w^{-1 / 2} \partial_{x}^{\alpha^{\prime}} h(s)\right\|_{Z_{q}}^{2}+\left\|w^{-1 / 2} \partial_{x}^{\alpha} h(s)\right\|^{2}\right) d s
\end{gathered}
$$

for any $t \geq 0$. Here, $w=w(\xi)$ is defined by

$$
w=w(\xi)= \begin{cases}1 & \text { for Model 1, } \\ 1+|\xi|^{2} & \text { for Model 2, } \\ 1+|\xi| & \text { for Model 3, }\end{cases}
$$

and the index $\sigma_{a, m}$ of the algebraic rate is defined by

$$
\sigma_{q, m}=\frac{d}{2}\left(\frac{1}{q}-\frac{1}{2}\right)+\frac{m}{2} .
$$

Case $\left(\Omega=\mathbb{T}^{d}\right)$. Suppose

$$
\iint_{\mathbb{T}^{d} \times \mathbb{R}^{d}} \psi(\xi) u_{0}(x, \xi) d x d \xi=0, \quad \forall \psi \in \text { ker L } .
$$

Then, there are constants $C$ and $\lambda>0$ such that

$$
\left\|e^{t \mathrm{~B}} u_{0}\right\| \leq C e^{-\lambda t}\left\|u_{0}\right\|
$$

for any $t \geq 0$. 
Theorem 1.2 (case of confining force). Consider 1.1) where $\Omega=\mathbb{R}^{d}, d \geq 1, V=V(x)$ is a confining force with $\int_{\mathbb{R}^{d}} e^{-V(x)} d x=1, \mathrm{~L}$ is one of the linear relaxation operator, linear FokkerPlanck operator and linearized Boltzmann operator as defined in Model 1, Model 2 and Model 3 , respectively. Let $e^{t \mathrm{~B}} u_{0}$ denote the corresponding solution for initial data $u_{0}=u_{0}(x, \xi)$. The following additional conditions on $V$ and $u_{0}$ are supposed to hold.

Case of Model 1: $V=\frac{|x|^{2}}{2}-\frac{d}{2} \ln (2 \pi)$, and

$$
\iint_{\mathbb{R}^{d} \times \mathbb{R}^{d}} \mathcal{M}^{1 / 2} u_{0} d x d \xi=0 .
$$

Case of Model 2:

$$
\begin{gathered}
\frac{1}{4}\left|\nabla_{x} V\right|^{2}-\frac{1}{2} \Delta_{x} V \rightarrow \infty \quad \text { as }|x| \rightarrow \infty \\
\left|\nabla_{x}^{2} V\right|^{2} \leq \delta\left|\nabla_{x} V\right|^{2}+C_{\delta}, \quad \forall \delta>0
\end{gathered}
$$

and

$$
\iint_{\mathbb{R}^{d} \times \mathbb{R}^{d}} \mathcal{M}^{1 / 2} u_{0} d x d \xi=0
$$

Case of Model 3: $V=\frac{|x|^{2}}{2}-\frac{d}{2} \ln (2 \pi), d \geq 3$, and

$$
\iint_{\mathbb{R}^{d} \times \mathbb{R}^{d}}\left(1, x, \xi, x \cdot \xi, x \times \xi,|x|^{2},|\xi|^{2}\right) \mathcal{M}^{1 / 2} u_{0} d x d \xi=0,
$$

where $(x \times \xi)_{i j}=x_{i} \xi_{j}-x_{j} \xi_{i}, 1 \leq i, j \leq d$.

Then, under the above assumptions, there are constants $C$ and $\lambda>0$ such that

$$
\left\|e^{t \mathrm{~B}}\right\|_{\mathcal{H}^{1}} \leq C e^{-\lambda t}\left\|u_{0}\right\|_{\mathcal{H}^{1}}
$$

for any $t \geq 0$.

The result for the case of Model 3 in Theorem 1.2 is new. To our knowledge, it is the first one dealing with the exponential rate for the linear Boltzmann equation with a confining force over the whole space. On the other hand, although other results stated in Theorem 1.1 and Theorem 1.2 have been extensively studied by many authors $[26,20,27,[16,15,17$, and $[23,28$, we here would give a self-contained proof by using an alternative unified approach for different models. Instead of the spectral analysis, compensation function and Lyapunov functionals in the previous work, the key part of our approach is to construct some equivalent temporal energy functionals for studying the time-decay property of solutions whether there is an external force or not and whether the spatial domain takes the whole space or torus. The essential point in the proof is using the macro-micro decomposition [22, 13, to reduce the problem to the analysis of the macroscopic fluid-type system which actually consists of evolution equations for moments of the kinetic equation.

1.6 Strategy of proof. As mentioned before, the main idea is the macro-micro decomposition combined with Kawashima's argument on dissipation of the hyperbolic-parabolic system. In fact, suppose that ker $\mathrm{L}$ is spanned by an orthogonal set $\mathcal{A}=\left\{\psi_{0}(\xi), \psi_{1}(\xi), \cdots, \psi_{n}(\xi)\right\}$ and the corresponding orthogonal velocity projection is denoted by

$$
\mathrm{P} u=\sum_{i=0}^{n} a_{i}(t, x) \psi_{i}(\xi)
$$


Hypocoercivity of Linear Degenerately Dissipative Kinetic Equations

The total energy dissipation rate corresponding to certain temporal energy functional of $u=$ $\{\mathrm{I}-\mathrm{P}\} u+\mathrm{P} u$ can be recovered as follows:

Step 1. Starting from the equation (1.1), one can make energy estimates to obtain the dissipation of the microscopic part $\{\mathrm{I}-\mathrm{P}\} u$ on the basis of the coercivity property 1.2 of the linearized operator L.

Step 2. One can derive a fluid-type one-order hyperbolic system of $a_{i}(t, x)(0 \leq i \leq n)$ coupled with $\{\mathrm{I}-\mathrm{P}\} u$ which are actually of the moment equations in terms of the above orthogonal set $\mathcal{A}$ and some high-order moment functions. See $(2.4),(2.16),(4.3)$ and $(4.17)$ for models under consideration. By applying Kawashima's argument on dissipation of the mixed hyperbolic-parabolic system [19], one can obtain the dissipation of the macroscopic part $\mathrm{P} u$ or equivalently $a_{i}(t, x)(0 \leq i \leq n)$ on the basis of the fluid-type system.

Step 3. Combining estimates in Step 1 and Step 2, one can obtain a properly defined temporal Lyapunov functional which is not only equivalent with the desired total energy functional but also captures the total energy dissipation rate.

Analytical tools are the Fourier transform for the case when there is no forcing and the direct energy estimates otherwise. When there is a potential force, we also need the Poincaré inequality and Korn-type inequality which will be provided at the last section.

\section{Relaxation model}

In this section we prove Theorem 1.1 and Theorem 1.2 for Model 1. Some similar results for this model are also obtained in [23, 15, 5].

2.1 Case when $\Omega=\mathbb{R}^{d}$ and $V=0$. To prove 1.14 and 1.15 , we consider the Cauchy problem

$$
\left\{\begin{array}{rlrl}
\partial_{t} u+\xi \cdot \nabla_{x} u & =\mathrm{L} u+h, & & t>0, x \in \mathbb{R}^{d}, \xi \in \mathbb{R}^{d}, \\
u(0, x, \xi) & =u_{0}(x, \xi), & x \in \mathbb{R}^{d}, \xi \in \mathbb{R}^{d}
\end{array}\right.
$$

where $\mathrm{L}$ is the relaxation operator given in $(1.4)$, and as in $(1.13), h=h(t, x, \xi)$ is orthogonal to ker L. Notice that the solution to 2.1 can be written as

$$
u(t)=e^{t \mathrm{~B}} u_{0}+\int_{0}^{t} e^{-(t-s) \mathrm{B}} h(s) d s,
$$

where $\mathrm{B}=-\xi \cdot \nabla_{x}+\mathrm{L}$.

We shall use the method of Fourier transform to deal with the time-decay of the solution $u$ given by 2.2 in a unifying manner so that 1.14 and 1.15 follow from the case when $h=0$ and $u_{0}=0$, respectively. In fact, the direct Fourier energy estimate on $(2.1)_{1}$ gives

$$
\partial_{t}\|\hat{u}\|_{L_{\xi}^{2}}^{2}+\left\|\left\{\mathrm{I}-\mathrm{P}_{0}\right\} \hat{u}\right\|_{L_{\xi}^{2}}^{2} \leq\|\hat{h}\|_{L_{\xi}^{2}}^{2}
$$

which is the first estimate on the basis of the dissipative property of L. Next, we estimate $\mathrm{P}_{0} u=a M^{1 / 2}$ with $a=a^{u}=\left\langle M^{1 / 2}, u\right\rangle$. Recall $b=b^{u}=\left\langle\xi M^{1 / 2}, u\right\rangle$. Then, from 2.1 $1, a$ and $b$ satisfy the fluid-type system

$$
\left\{\begin{array}{l}
\partial_{t} a+\nabla_{x} \cdot b=0 \\
\partial_{t} b+\nabla_{x} a+\nabla_{x} \cdot \Gamma\left(\left\{\mathrm{I}-\mathrm{P}_{0}\right\} u\right)=-b+\left\langle\xi M^{1 / 2}, h\right\rangle,
\end{array}\right.
$$


where $\Gamma=\left(\Gamma_{i j}\right)_{d \times d}$ is the moment function defined by

$$
\Gamma_{i j}(g)=\left\langle\left(\xi_{i} \xi_{j}-1\right) M^{1 / 2}, g\right\rangle, \quad 1 \leq i, j \leq d .
$$

Notice by the definition of $\mathrm{P}_{0}$ that $\Gamma\left(\left\{\mathrm{I}-\mathrm{P}_{0}\right\} u\right)=\left\langle\xi \otimes \xi M^{1 / 2},\left\{\mathrm{I}-\mathrm{P}_{0}\right\} u\right\rangle$. Here, we used 2.5 as the definition of $\Gamma$ only for the convenience of the later proof for Model 3. We now make estimates on the Fourier transform of (2.4). In fact, as in [9], due to

$$
\begin{aligned}
|k|^{2}|\hat{a}|^{2}= & (\mathrm{i} k \hat{a} \mid \mathrm{i} k \hat{a})=\left(\mathrm{i} k \hat{a} \mid-\partial_{t} \hat{b}-\mathrm{i} \Gamma\left(\left\{\mathrm{I}-\mathrm{P}_{0}\right\} \hat{u}\right) \cdot k-\hat{b}+\left\langle\xi M^{1 / 2}, \hat{h}\right\rangle\right) \\
= & -\partial_{t}(\mathrm{i} \hat{a} \mid \hat{b})+|k \cdot \hat{b}|^{2} \\
& +\left(\mathrm{i} k \hat{a} \mid-\mathrm{i} \Gamma\left(\left\{\mathrm{I}-\mathrm{P}_{0}\right\} \hat{u}\right) \cdot k-\hat{b}+\left\langle\xi M^{1 / 2}, \hat{h}\right\rangle\right),
\end{aligned}
$$

one has by further using Cauchy-Schwarz and dividing it by $1+|k|^{2}$,

$$
\partial_{t} \operatorname{Re} \frac{(\mathrm{i} \hat{a} \mid \hat{b})}{1+|k|^{2}}+\frac{\lambda|k|^{2}}{1+|k|^{2}}|\hat{a}|^{2} \leq C\left(\left\|\left\{I-\mathrm{P}_{0}\right\} \hat{u}\right\|_{L_{\xi}^{2}}^{2}+\|\hat{h}\|_{L_{\xi}^{2}}^{2}\right),
$$

which is the second estimate based on the Kawashima's argument on the dissipation of the hyperbolic-parabolic system.

Now, for $t \geq 0, k \in \mathbb{R}^{d}$, define

$$
E(\hat{u})=\|\hat{u}\|_{L_{\xi}^{2}}^{2}+\kappa \operatorname{Re} \frac{(\mathrm{i} \hat{a} \mid \hat{b})}{1+|k|^{2}}
$$

with a small constant $\kappa>0$ to be determined later. One can let $\kappa>0$ be small such that $E(\hat{u}) \sim\|\hat{u}\|_{L_{\xi}^{2}}^{2}$. Taking $\kappa>0$ further small, the linear combination of 2.3) and 2.6) gives

$$
\partial_{t} E(\hat{u})+\frac{\lambda|k|^{2}}{1+|k|^{2}} E(\hat{u}) \leq C\|\hat{h}\|_{L_{\xi}^{2}}^{2}
$$

which with the help of Gronwall's inequality implies

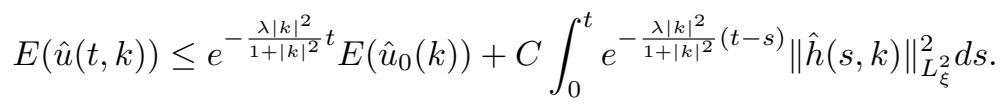

Hence, (1.14) and 1.15 follows from the above estimate by using the standard procedure as in 19, 11, 27, and the details of the rest proof are omitted for simplicity. This completes the proof of 1.14 and 1.15 for Model 1 in Theorem 1.1 .

2.2 Case when $\Omega=\mathbb{T}^{d}$ and $V=0$. The goal in this case is to prove (1.17). It actually can be achieved by a little modification in the case of the whole space. In fact, let's consider the Cauchy problem 2.1 with the spatial domain $\mathbb{R}^{d}$ replaced by $\mathbb{T}^{d}$ and $h=0$. Then, 2.3 and (2.6) with $h=0$ still hold for $\Omega=\mathbb{T}^{d}$. $a$ and $b$ still satisfy the system (2.4). Notice that due to the mass conservation and the assumption 1.16 , one has $\int_{\mathbb{T}^{d}} a(t, x) d x \equiv 0$ and hence $\hat{a}(t, 0)=0$ for any $t \geq 0$. Therefore, 2.6 is modified as

$$
\partial_{t} \operatorname{Re} \frac{(\mathrm{i} \hat{a} \mid \hat{b})}{1+|k|^{2}}+\lambda|\hat{a}|^{2} \leq C\left\|\left\{\mathrm{I}-\mathrm{P}_{0}\right\} \hat{u}\right\|_{L_{\xi}^{2}}^{2} .
$$

By using the same definition of $E(\hat{u})$ as in $(2.7)$, it holds that $\partial_{t} E(\hat{u})+\lambda E(\hat{u}) \leq 0$, which implies 1.17 from Gronwall's inequality and $k$-integration. This completes the proof of 1.17) for Model 1 in Theorem 1.1. 
2.3 Case when $\Omega=\mathbb{R}^{d}$ and $V$ is confining. In particular, let $V=\frac{|x|^{2}}{2}-\frac{d}{2} \ln (2 \pi)$. Take $u_{0}$ with

$$
\iint_{\mathbb{T}^{d} \times \mathbb{R}^{d}} \mathcal{M}^{1 / 2} u_{0} d x d \xi=0
$$

Let $u(t)=e^{t \mathrm{~B}} u_{0}$ be the solution to the Cauchy problem

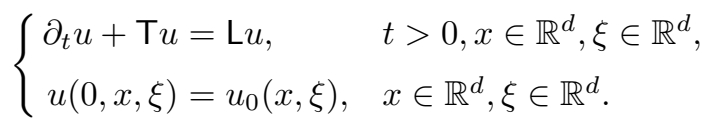

Next, we make energy estimates on $u$. For zero-order, it is straightforward to get

$$
\frac{1}{2} \frac{d}{d t}\|u\|^{2}+\left\|\left\{I-\mathrm{P}_{0}\right\} u\right\|^{2}=0 .
$$

For first-order, instead of directly estimating $x$ and $\xi$ derivatives, we use $\mathrm{X}$ and $\mathrm{Y}$ differentiations. Take $1 \leq i \leq d$. Applying $\mathrm{X}_{i}$ and $\mathrm{Y}_{i}$ to $(2.9)_{1}$, one has

$$
\begin{aligned}
& \partial_{t} \mathrm{X}_{i} u+\mathrm{TX}_{i} u-\mathrm{LX}_{i} u=\left[\mathrm{T}, \mathrm{X}_{i}\right] u-\left[\mathrm{L}, \mathrm{X}_{i}\right] u, \\
& \partial_{t} \mathrm{Y}_{i} u+\mathrm{TY}_{i} u-\mathrm{LY}_{i} u=\left[\mathrm{T}, \mathrm{Y}_{i}\right] u-\left[\mathrm{L}, \mathrm{Y}_{i}\right] u,
\end{aligned}
$$

where $[\cdot, \cdot]$ denotes commutator of two operators. Since $\mathrm{L}$ is local in $x$ and $\mathrm{X}_{i}$ is only involved in spatial derivative and multiplier, then $\left[\mathrm{L}, \mathrm{X}_{i}\right]=0$. Due to the fact that $\mathrm{P}_{0} \mathrm{Y}_{i}=\mathrm{Y}_{i} \mathrm{P}_{0}=0$,

$$
\left[\mathrm{L}, \mathrm{Y}_{i}\right]=\left[\mathrm{I}-\mathrm{P}_{0}, \mathrm{Y}_{i}\right]=-\left[\mathrm{P}_{0}, \mathrm{Y}_{i}\right]=0 .
$$

For commutators containing $\mathrm{T}$ in 2.11 and 2.12 , the further computations yield

$$
\left[\mathrm{T}, \mathrm{X}_{i}\right] u=\nabla_{x} \partial_{i} V \cdot \mathrm{Y} u, \quad\left[\mathrm{~T}, \mathrm{Y}_{i}\right] u=-\mathrm{X}_{i} u .
$$

Thus, by noticing $\partial_{i} \partial_{j} V=\delta_{i j}$ by the definition of $V$ in the considered case, it follows from (2.11) and 2.12) that

$$
\frac{1}{2} \frac{d}{d t}\left(\|\mathrm{X} u\|^{2}+\|\mathrm{Y} u\|^{2}\right)+\left\|\left\{\mathrm{I}-\mathrm{P}_{0}\right\} \mathrm{X} u\right\|^{2}+\left\|\left\{\mathrm{I}-\mathrm{P}_{0}\right\} \mathrm{Y} u\right\|^{2}=0 .
$$

Since $\mathrm{P}_{0} \mathrm{Y}=0$, the rest is to obtain the dissipation rate corresponding to $\mathrm{P}_{0} \mathrm{X} u=\mathrm{XP}_{0} u$ and $\mathrm{P}_{0} u$, or equivalently $\mathrm{X} a$ and $a$. We shall again turn to the fluid-type system satisfied by $a$ and $b$.

Notice that when $V$ is nontrivial, similarly as before, from $2.9{ }_{1}, a$ and $b$ satisfy

$$
\left\{\begin{array}{l}
\partial_{t}\left(a e^{-\frac{V}{2}}\right)+\nabla_{x} \cdot\left(b e^{-\frac{V}{2}}\right)=0, \\
\partial_{t}\left(b e^{-\frac{V}{2}}\right)+\nabla_{x}\left(a e^{-\frac{V}{2}}\right)+\nabla_{x} V a e^{-\frac{V}{2}}+\nabla_{x} \cdot\left[\Gamma\left(\left\{\mathrm{I}-\mathrm{P}_{0}\right\} u\right) e^{-\frac{V}{2}}\right]=-b e^{-\frac{V}{2}} .
\end{array}\right.
$$

Equivalently, the above system can be rewritten as

$$
\left\{\begin{array}{l}
\partial_{t} a-\mathbf{X}^{*} \cdot b=0 \\
\partial_{t} b+\mathrm{X} a-\mathbf{X}^{*} \cdot \Gamma\left(\left\{\mathrm{I}-\mathrm{P}_{0}\right\} u\right)+b=0
\end{array}\right.
$$

where $\mathrm{X}^{*}$ is the adjoint operator of $\mathrm{X}$ given by

$$
\mathrm{X}_{i}^{*} u=-e^{\frac{V(x)}{2}} \partial_{x_{i}}\left(e^{-\frac{V(x)}{2}} u\right)=\left(\frac{1}{2} \partial_{x_{i}} V-\partial_{x_{i}}\right) u, \quad 1 \leq i \leq d .
$$


Noticing that 2.8 together with the mass conservation $2.15{ }_{1}$ imply

$$
\left.\int_{\mathbb{R}^{d}} e^{-\frac{V(x)}{2}} a(t, x) d x\right|_{t \geq 0}=0 .
$$

From $(2.16)_{2}$, one can compute

$$
\begin{aligned}
\|\mathrm{X} a\|^{2} & =(\mathrm{X} a, \mathrm{X} a)=\left(\mathrm{X} a,-\partial_{t} b+\mathrm{X}^{*} \cdot \Gamma\left(\left\{\mathrm{I}-\mathrm{P}_{0}\right\} u\right)-b\right) \\
& =-\frac{d}{d t}(\mathrm{X} a, b)+\left(\mathrm{X} \partial_{t} a, b\right)+\left(\mathrm{X} a, \mathbf{X}^{*} \cdot \Gamma\left(\left\{\mathrm{I}-\mathrm{P}_{0}\right\} u\right)-b\right)
\end{aligned}
$$

where it further holds from $2.16{ }_{1}$ that

$$
\left(\mathbf{X} \partial_{t} a, b\right)=\left(\partial_{t} a, \mathbf{X}^{*} \cdot b\right)=\left(\mathbf{X}^{*} \cdot b, \mathbf{X}^{*} \cdot b\right)=\left\|\mathbf{X}^{*} \cdot b\right\|^{2} .
$$

Then, it follows that

$$
\frac{d}{d t}(\mathrm{X} a, b)+\lambda\|\mathrm{X} a\|^{2} \leq\left\|\mathbf{X}^{*} \cdot b\right\|^{2}+C\left(\left\|\mathbf{X}^{*}\left\{\mathrm{I}-\mathrm{P}_{0}\right\} u\right\|^{2}+\|b\|^{2}\right) .
$$

Lemma 2.1. As long as there is a constant $C$ such that

$$
\left|\Delta_{x} V\right|^{2} \leq C\left(\left|\nabla_{x} V\right|^{2}+1\right)
$$

for all $x \in \mathbb{R}^{d}$, there is some constant $C$ such that

$$
\int_{\mathbb{R}^{d}}\left|\mathbf{X}^{*} g\right|^{2} d x \leq C \int_{\mathbb{R}^{d}}\left(|\mathbf{X} g|^{2}+|g|^{2}\right) d x
$$

for $g=g(x)$.

Proof. From integration by parts,

$$
\int_{\mathbb{R}^{d}}\left|\mathbf{X}^{*} g\right|^{2} d x=\int_{\mathbb{R}^{d}}|\mathrm{X} g|^{2} d x+\int_{\mathbb{R}^{d}}|g|^{2} \Delta_{x} V d x .
$$

By further applying Cauchy-Schwarz to $\Delta_{x} V$ in the above equation and then using the assumption 2.19), one can show that

$$
\int_{\mathbb{R}^{d}}|g|^{2} \Delta_{x} V d x \leq C \int_{\mathbb{R}^{d}}|\mathrm{X} g|^{2} d x+C \int_{\mathbb{R}^{d}}|g|^{2} d x .
$$

2.20 follows by plugging the above inequality into 2.21.

So, by applying Lemma 2.1 to 2.18), one has

$$
\frac{d}{d t}(\mathrm{X} a, b)+\lambda\|\mathrm{X} a\|^{2} \leq C\left(\left\|\left\{\mathrm{I}-\mathrm{P}_{0}\right\} \mathrm{X} u\right\|^{2}+\left\|\left\{\mathrm{I}-\mathrm{P}_{0}\right\} u\right\|^{2}\right) .
$$

Furthermore, due to (2.17), one has Poincaré inequality $\|\mathrm{X} a\|^{2} \geq \lambda\|a\|^{2}$ from Proposition 5.1 Then, it follows that

$$
\frac{d}{d t}(\mathrm{X} a, b)+\lambda\left(\|\mathrm{X} a\|^{2}+\|a\|^{2}\right) \leq C\left(\left\|\left\{\mathrm{I}-\mathrm{P}_{0}\right\} \mathbf{X} u\right\|^{2}+\left\|\left\{\mathrm{I}-\mathrm{P}_{0}\right\} u\right\|^{2}\right) .
$$


Now, let us define a temporal functional

$$
\mathcal{E}(u(t))=\|u\|^{2}+\|\mathrm{X} u\|^{2}+\|\mathrm{Y} u\|^{2}+\kappa(\mathrm{X} a, b)
$$

with a small constant $\kappa>0$ to be determined. Firstly, $\kappa>0$ is chosen small such that $\mathcal{E}(u(t)) \sim\|u(t)\|_{\mathcal{H}^{1}}^{2}$. And then, $\kappa>0$ is further small enough such that the linear combination of (2.10, 2.14 and 2.22) gives

$$
\frac{d}{d t} \mathcal{E}(u(t))+\lambda \mathcal{E}(u(t)) \leq 0
$$

By Gronwall's inequality, this proves 1.18 and hence completes the proof of Theorem 1.2 for Model 1.

\section{Fokker-Planck equation}

In this section we prove Theorem 1.1 and Theorem 1.2 for Model 2. The proof in the case of no forcing can be carried out in the completely same way as for Model 1. When a stationary potential forcing is present, the proof for Model 1 can be refined to yield the exponential time-decay rate in $\mathcal{H}^{1}$ even for a class of general potential functions essentially because the Fokker-Planck operator enjoys the velocity regularity. This actually has been studied in detail by Villani [28, and the hypoelliptic theory is founded by Hérau [15, 17]. See also [23, 8] and references therein. Here, we shall give another proof which is based on the macro-micro decomposition and Kawashima's dissipation argument on the hyperbolic-parabolic system.

3.1 Case when $\Omega=\mathbb{R}^{d}$ or $\mathbb{T}^{d}$ and $V=0$. We consider the Cauchy problem

$$
\left\{\begin{aligned}
\partial_{t} u+\xi \cdot \nabla_{x} u & =\mathrm{L} u+h, & & t>0, x \in \Omega, \xi \in \mathbb{R}^{d}, \\
u(0, x, \xi) & =u_{0}(x, \xi), & & x \in \Omega, \xi \in \mathbb{R}^{d},
\end{aligned}\right.
$$

where $\mathrm{L}$ is the self-adjoint Fokker-Planck operator given in $(1.6)$, and as in $(1.13), h=h(t, x, \xi)$ is orthogonal to ker L. The solution $u(t)$ to 3.1 can be written as the same form as in $(2.2)$. In this case, Theorem 1.1 and Theorem 1.2 for Model 2 can be proved in the same way as for Model 1. Thus, all details of the proof in this case are omitted for simplicity.

3.2 Case when $\Omega=\mathbb{R}^{d}$ and $V$ is confining. Suppose

$$
\begin{gathered}
\int_{\mathbb{R}^{d}} e^{-V(x)} d x=1, \\
\frac{1}{4}\left|\nabla_{x} V\right|^{2}-\frac{1}{2} \Delta_{x} V \rightarrow \infty \quad \text { as } \quad|x| \rightarrow \infty, \\
\left|\nabla_{x}^{2} V\right| \leq \delta\left|\nabla_{x} V\right|^{2}+C_{\delta}, \quad \forall \delta>0 .
\end{gathered}
$$

Take $u_{0}$ with

$$
\iint_{\mathbb{T}^{d} \times \mathbb{R}^{d}} \mathcal{M}^{1 / 2} u_{0} d x d \xi=0
$$

Let $u(t)=e^{t \mathrm{~B}} u_{0}$ be the solutio to the Cauchy problem

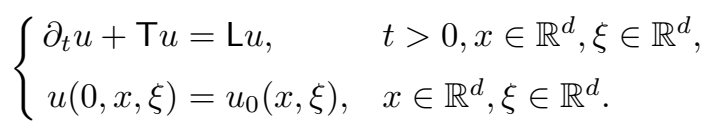


Next, we follow the same line of proof as for Model 1. It is straightforward to get from (3.6) 1 that

$$
\frac{1}{2} \frac{d}{d t}\|u\|^{2}+\lambda\left\|\left\{\mathrm{I}-\mathrm{P}_{0}\right\} u\right\|_{\nu}^{2} \leq 0
$$

where $\nu=\nu(\xi)=1+|\xi|^{2}$. Recall the definition 1.30 of the operator $\mathrm{Y}$. Observe that since $\mathrm{L} u=\Delta_{\xi} u+\frac{1}{4}\left(2 d-|\xi|^{2}\right) u$, then $[\mathrm{L}, \mathrm{Y}]=\mathrm{Y}$. Thus, similar to obtain 2.11-2.12 with the identity 2.13, one has

$$
\begin{gathered}
\frac{1}{2} \frac{d}{d t}\|\mathrm{X} u\|^{2}+\lambda\left\|\left\{\mathrm{I}-\mathrm{P}_{0}\right\} \mathrm{X} u\right\|_{\nu}^{2} \leq \sum_{i j=1}^{d} \iint_{\mathbb{R}^{d} \times \mathbb{R}^{d}} \partial_{i} \partial_{j} V \mathrm{X}_{i} u \mathrm{Y}_{j} u d x d \xi, \\
\frac{1}{2} \frac{d}{d t}\|\mathrm{Y} u\|^{2}+\lambda\left\|\left\{\mathbf{I}-\mathrm{P}_{0}\right\} \mathrm{Y} u\right\|_{\nu}^{2}+\|\mathrm{Y} u\|^{2} \leq-\sum_{i=1}^{d} \iint_{\mathbb{R}^{d} \times \mathbb{R}^{d}} \mathrm{X}_{i} u \mathrm{Y}_{j} u d x d \xi .
\end{gathered}
$$

The r.h.s. of $(3.8)$ is bounded as

$$
\begin{aligned}
& \sum_{i j=1}^{d} \iint_{\mathbb{R}^{d} \times \mathbb{R}^{d}} \partial_{i} \partial_{j} V \mathrm{X}_{i} u \mathrm{Y}_{j} u d x d \xi \\
& =\sum_{i j=1}^{d} \iint_{\mathbb{R}^{d} \times \mathbb{R}^{d}} \partial_{i} \partial_{j} V \mathrm{Y}_{j}^{*}\left\{\mathrm{I}-\mathrm{P}_{0}\right\} \mathbf{X}_{i} u\left\{\mathbf{I}-\mathrm{P}_{0}\right\} u d x d \xi \\
& \leq \epsilon\left\|\left\{\mathbf{I}-\mathrm{P}_{0}\right\} \mathbf{X} u\right\|_{\nu}^{2}+\frac{C}{\epsilon} \iint_{\mathbb{R}^{d} \times \mathbb{R}^{d}}\left|\nabla_{x}^{2} V\right|^{2}\left|\left\{\mathbf{I}-\mathrm{P}_{0}\right\} u\right|^{2} d x d \xi
\end{aligned}
$$

for an arbitrary constant $\epsilon>0$ to be chosen later, where we used $\mathrm{YP}_{0} u=0$ and $\mathrm{Y}^{*}$ is the adjoint operator of $\mathrm{Y}$ given by $\mathrm{Y}_{j}^{*} u=\left(\frac{1}{2} \xi_{j}-\partial_{\xi_{j}}\right) u$. Furthermore, due to the assumption 3.4. on $V$,

$$
\iint_{\mathbb{R}^{d} \times \mathbb{R}^{d}}\left|\nabla_{x}^{2} V\right|^{2}\left|\left\{\mathrm{I}-\mathrm{P}_{0}\right\} u\right|^{2} d x d \xi \leq C \delta\left\|\left\{\mathrm{I}-\mathrm{P}_{0}\right\} \mathrm{X} u\right\|^{2}+C_{\delta}\left\|\left\{\mathrm{I}-\mathrm{P}_{0}\right\} u\right\|^{2} .
$$

So, from the above estimates, by choosing $\epsilon>0$ and $\delta>0$ small enough, 3.8 is bounded by

$$
\frac{1}{2} \frac{d}{d t}\|\mathrm{X} u\|^{2}+\lambda\left\|\left\{\mathrm{I}-\mathrm{P}_{0}\right\} \mathbf{X} u\right\|_{\nu}^{2} \leq C\left\|\left\{\mathrm{I}-\mathrm{P}_{0}\right\} u\right\|^{2} .
$$

Noticing $\left\langle\mathrm{X}_{i} u, \mathrm{Y}_{i} u\right\rangle=\left\langle\left\{\mathrm{I}-\mathrm{P}_{0}\right\} \mathrm{X}_{i} u, \mathrm{Y}_{i} u\right\rangle$, one has from (3.9) that

$$
\frac{1}{2} \frac{d}{d t}\|\mathrm{Y} u\|^{2}+\lambda\left\|\left\{\mathrm{I}-\mathrm{P}_{0}\right\} \mathrm{Y} u\right\|_{\nu}^{2} \leq C\left\|\left\{\mathrm{I}-\mathrm{P}_{0}\right\} \mathrm{X} u\right\|^{2} .
$$

Finally, we turn to the estimate on $\mathrm{P}_{0} \mathrm{X} u$ and $\mathrm{P}_{0} u$. Similar to obtain 2.16, $3.6{ }_{1}$ also gives the same fluid-type system:

$$
\left\{\begin{array}{l}
\partial_{t} a-\mathbf{X}^{*} \cdot b=0 \\
\partial_{t} b+\mathrm{X} a-\mathbf{X}^{*} \cdot \Gamma\left(\left\{I-\mathrm{P}_{0}\right\} u\right)+b=0
\end{array}\right.
$$

with

$$
\left.\int_{\mathbb{R}^{d}} e^{-\frac{V(x)}{2}} a(t, x) d x\right|_{t \geq 0}=0
$$


again due to the mass conservation and initial condition 3.5). Applying the same argument as before and then using Poincaré inequality in Proposition 5.1 by assumptions (3.2)- 3.3 , one has

$$
\frac{d}{d t}(\mathrm{X} a, b)+\lambda\left(\|\mathrm{X} a\|^{2}+\|a\|^{2}\right) \leq C\left(\left\|\left\{\mathbf{I}-\mathrm{P}_{0}\right\} \mathbf{X} u\right\|^{2}+\left\|\left\{\mathrm{I}-\mathrm{P}_{0}\right\} u\right\|^{2}\right) .
$$

Now, let us define a temporal functional

$$
\mathcal{E}(u(t))=\|u\|^{2}+\kappa_{1}\|\mathrm{X} u\|^{2}+\kappa_{2}\|\mathrm{Y} u\|^{2}+\kappa_{3}(\mathrm{X} a, b)
$$

with constants $\kappa_{i}>0(1 \leq i \leq 3)$. One can choose $0<\kappa_{3} \ll \kappa_{2} \ll \kappa_{1} \ll 1$ such that $\mathcal{E}(u(t)) \sim\|u(t)\|_{\mathcal{H}^{1}}^{2}$ and further the linear combination of (3.7), 3.10, 3.11) and 3.12 gives

$$
\frac{d}{d t} \mathcal{E}(u(t))+\lambda \mathcal{E}(u(t)) \leq 0
$$

By Gronwall's inequality, this proves (1.18) and hence completes the proof of Theorem 1.2 for Model 2.

\section{Boltzmann equation}

In this section we prove Theorem 1.1 and Theorem 1.2 for Model 3. Although this can be done along the same line as for Model 1 and Model 2, it is a little more complicated for Model 3 since ker $\mathbf{L}=d+2$ so that the linearized Boltzmann operator $\mathbf{L}$ is degenerate over a space with higher dimensions. The key idea is still based on the macro-micro decomposition [14, 22, 21] and Kawashima's dissipation argument [19] on the hyperbolic-parabolic system. The additional difficulty in the presence of confining forces lies in verifying the Korn-type inequality to obtain the dissipation of momentum component in the fluid part, which is left to the next section. For the application of the classical Korn inequality to the Boltzmann equation or the fluid dynamic equations, refer to [4] and 28.

4.1 Case when $\Omega=\mathbb{R}^{d}$ and $V=0$. We consider the Cauchy problem

$$
\left\{\begin{array}{rlrl}
\partial_{t} u+\xi \cdot \nabla_{x} u & =\mathrm{L} u+h, & & t>0, x \in \mathbb{R}^{d}, \xi \in \mathbb{R}^{d}, \\
u(0, x, \xi) & =u_{0}(x, \xi), \quad x \in \mathbb{R}^{d}, \xi \in \mathbb{R}^{d},
\end{array}\right.
$$

where $\mathbf{L}$ is the linearized Boltzmann collision operator given in (1.8), and as in 1.13$), h=$ $h(t, x, \xi)$ is orthogonal to ker $\mathrm{L}$. The solution $u(t)$ to 4.1 can be written as the same form as in 2.2 . $u(t)$ will be estimated by using the method of Fourier transform similarly before.

Firstly, from $4.11_{1}$, it is straightforward to obtain

$$
\frac{1}{2} \partial_{t}\|\hat{u}\|_{L_{\xi}^{2}}^{2}+\lambda \iint_{\mathbb{R}^{d} \times \mathbb{R}^{d}} \nu(\xi)|\{\mathbf{I}-\mathrm{P}\} \hat{u}|^{2} d x d \xi \leq C\left\|\nu^{-1 / 2} \hat{h}\right\|_{L_{\xi}^{2}}^{2},
$$

where $\nu(\xi) \sim 1+|\xi|$ defined by 11.10 is the collision frequency for the case of hard sphere model [11, and the orthogonal velocity projection operator $\mathrm{P}: L_{\xi}^{2} \rightarrow \operatorname{ker} \mathrm{L}$ is described by (1.11). Next, we devote ourselves to the estimate on $\mathrm{P} u$ or equivalently $(a, b, c)$. In fact, by taking $\frac{1}{2} d(d+5)+2$ number of velocity moments

$$
M^{1 / 2}, \xi_{i} M^{1 / 2},\left(|\xi|^{2}-d\right) M^{1 / 2},\left(\xi_{i} \xi_{j}-1\right) M^{1 / 2},\left(|\xi|^{2}-d-2\right) \xi_{i} M^{1 / 2}
$$


with $1 \leq i, j \leq d$ for the equation 4.11 , one has the fluid-type system

$$
\left\{\begin{array}{l}
\partial_{t} a+\nabla_{x} \cdot b=0, \\
\partial_{t} b+\nabla_{x}(a+2 c)+\nabla_{x} \cdot \Gamma(\{\mathrm{I}-\mathrm{P}\} u)=0, \\
\partial_{t} c+\frac{1}{d} \nabla_{x} \cdot b+\frac{1}{2 d} \nabla_{x} \cdot \Lambda(\{\mathrm{I}-\mathrm{P}\} u)=0, \\
\partial_{t}\left[\Gamma_{i j}(\{\mathrm{I}-\mathrm{P}\} u)+2 c \delta_{i j}\right]+\partial_{i} b_{j}+\partial_{j} b_{i}=\Gamma_{i j}(r+h), \\
\partial_{t} \Lambda_{i}(\{\mathrm{I}-\mathrm{P}\} u)+\partial_{i} c=\Lambda_{i}(r+h),
\end{array}\right.
$$

where $a, b, c$ are defined in (1.11), the matrix-valued function $\Gamma$ is defined by (2.5), the moment function $\Lambda=\left(\Lambda_{i}\right)_{1 \leq i \leq d}$ is defined by

$$
\Lambda_{i}(g)=\left\langle\left(|\xi|^{2}-d-2\right) \xi_{i} M^{1 / 2}, g\right\rangle,
$$

and $r$ is denoted by $r=-\xi \cdot \nabla_{x}\{\mathrm{I}-\mathrm{P}\} u+\mathrm{L}\{\mathrm{I}-\mathrm{P}\} u$. The detailed derivation of 4.3 was given in [7] and thus is omitted for simplicity.

Lemma 4.1. There exists a functional $E_{\text {int }}(\hat{u})$ given by

$$
\begin{aligned}
E_{\text {int }}(\hat{u})= & \frac{1}{1+|k|^{2}} \sum_{i=1}^{d}\left(\mathrm{i} k_{i} \hat{c} \mid \Lambda_{i}(\{\mathrm{I}-\mathrm{P}\} \hat{u})\right) \\
& +\frac{\kappa_{1}}{1+|k|^{2}} \sum_{i j=1}^{d}\left(\mathrm{i} k_{i} \hat{b}_{j}+\mathrm{i} k_{j} \hat{b}_{i} \mid \Gamma_{i j}(\{\mathrm{I}-\mathrm{P}\} \hat{u})+2 \hat{c} \delta_{i j}\right) \\
& +\frac{\kappa_{2}}{1+|k|^{2}} \sum_{i=1}^{d}\left(\mathrm{i} k_{i} \hat{a} \mid \hat{b}_{i}\right)
\end{aligned}
$$

for constants $0<\kappa_{2} \ll \kappa_{1} \ll 1$ such that there are constants $\lambda>0, C$ such that

$$
\partial_{t} \operatorname{Re} E_{\text {int }}(\hat{u})+\frac{\lambda|k|^{2}}{1+|k|^{2}}\left(|\hat{a}|^{2}+|\hat{b}|^{2}+|\hat{c}|^{2}\right) \leq C\left(\|\{\mathrm{I}-\mathrm{P}\} \hat{u}\|_{L_{\xi}^{2}}^{2}+\left\|\nu^{-1 / 2} \hat{h}\right\|_{L_{\xi}^{2}}^{2}\right)
$$

holds for any $t \geq 0$ and $k \in \mathbb{R}^{d}$.

Proof. Write 4.3$]$ in terms of Fourier transform as

$$
\left\{\begin{array}{l}
\partial_{t} \hat{a}+\mathrm{i} k \cdot \hat{b}=0, \\
\partial_{t} \hat{b}+\mathrm{i}(\hat{a}+2 \hat{c})+\mathrm{i} \Gamma(\{\mathrm{I}-\mathrm{P}\} \hat{u}) \cdot k=0, \\
\partial_{t} \hat{c}+\frac{1}{d} \mathrm{i} k \cdot \hat{b}+\frac{1}{2 d} \mathrm{i} k \cdot \Lambda(\{\mathrm{I}-\mathrm{P}\} \hat{u})=0, \\
\partial_{t}\left[\Gamma_{i j}(\{\mathrm{I}-\mathrm{P}\} \hat{u})+2 \hat{c} \delta_{i j}\right]+\mathrm{i} k_{i} \hat{b}_{j}+\mathrm{i} k_{j} \hat{b}_{i}=\Gamma_{i j}(\hat{r}+\hat{h}), \\
\partial_{t} \Lambda_{i}(\{\mathrm{I}-\mathrm{P}\} \hat{u})+\mathrm{i} k_{i} \hat{c}=\Lambda_{i}(\hat{r}+\hat{h}),
\end{array}\right.
$$

for $1 \leq i, j \leq d$, where $\hat{r}=-\mathrm{i} \xi \cdot k\{\mathrm{I}-\mathrm{P}\} \hat{u}+\mathrm{L}\{\mathrm{I}-\mathrm{P}\} \hat{u}$. 
Step 1. Estimate $\hat{c}$ : Starting from the highest-order moment equation $4.7{ }_{5}$, one has

$$
\begin{aligned}
|k|^{2}|\hat{c}|^{2}= & \sum_{i=1}^{d}\left(\mathrm{i} k_{i} \hat{c} \mid \mathrm{i} k_{i} \hat{c}\right)=\sum_{i=1}^{d}\left(\mathrm{i} k_{i} \hat{c} \mid-\partial_{t} \Lambda_{i}(\{\mathrm{I}-\mathrm{P}\} \hat{u})+\Lambda_{i}(\hat{r}+\hat{h})\right) \\
= & -\partial_{t} \sum_{i=1}^{d}\left(\mathrm{i} k_{i} \hat{c} \mid \Lambda_{i}(\{\mathrm{I}-\mathrm{P}\} \hat{u})\right)+\sum_{i=1}^{d}\left(\mathrm{i} k_{i} \partial_{t} \hat{c} \mid \Lambda_{i}(\{\mathrm{I}-\mathrm{P}\} \hat{u})\right) \\
& +\sum_{i=1}^{d}\left(\mathrm{i} k_{i} \hat{c} \mid \Lambda_{i}(\hat{r}+\hat{h})\right) .
\end{aligned}
$$

Using 4.7$]_{3}$ to replace $\partial_{t} \hat{c}$ and then using Cauchy-Schwarz, it follows

$$
\begin{aligned}
& \partial_{t} \operatorname{Re} \sum_{i=1}^{d}\left(\mathrm{i} k_{i} \hat{c} \mid \Lambda_{i}(\{\mathrm{I}-\mathrm{P}\} \hat{u})\right)+\lambda|k|^{2}|\hat{c}|^{2} \\
& \quad \leq \epsilon_{1}|k \cdot \hat{b}|^{2}+\frac{C}{\epsilon_{1}}\left(1+|k|^{2}\right)\|\{\mathrm{I}-\mathrm{P}\} \hat{u}\|_{L_{\xi}^{2}}^{2}+C\left\|\nu^{-1 / 2} \hat{h}\right\|_{L_{\xi}^{2}}^{2},
\end{aligned}
$$

where $0<\epsilon_{1} \leq 1$ is arbitrary to be chosen later.

Step 2. Estimate $\hat{b}$ : Observe the identity

$$
\sum_{i j=1}^{d}\left|\mathrm{i} k_{i} \hat{b}_{j}+\mathrm{i} k_{j} \hat{b}_{i}\right|^{2}=2|k|^{2}|\hat{b}|^{2}+2|k \cdot \hat{b}|^{2} .
$$

On the other hand, compute from 4.7$]_{4}$ that

$$
\begin{aligned}
& \sum_{i j=1}^{d}\left\|\mathrm{i} k_{i} \hat{b}_{j}+\mathrm{i} k_{j} \hat{b}_{i}\right\|^{2}=\sum_{i j=1}^{d}\left(\mathrm{i} k_{i} \hat{b}_{j}+\mathrm{i} k_{j} \hat{b}_{i} \mid-\partial_{t}\left[\Gamma_{i j}(\{\mathrm{I}-\mathrm{P}\} \hat{u})+2 \hat{c} \delta_{i j}\right]+\Gamma_{i j}(\hat{r}+\hat{h})\right) \\
& =-\partial_{t} \sum_{i j=1}^{d}\left(\mathrm{i} k_{i} \hat{b}_{j}+\mathrm{i} k_{j} \hat{b}_{i} \mid \Gamma_{i j}(\{\mathrm{I}-\mathrm{P}\} \hat{u})+2 \hat{c} \delta_{i j}\right) \\
& \quad+\sum_{i j=1}^{d}\left(\mathrm{i} k_{i} \partial_{t} \hat{b}_{j}+\mathrm{i} k_{j} \partial_{t} \hat{b}_{i} \mid \Gamma_{i j}(\{\mathrm{I}-\mathrm{P}\} \hat{u})+2 \hat{c} \delta_{i j}\right)+\sum_{i j=1}^{d}\left(\mathrm{i} k_{i} \hat{b}_{j}+\mathrm{i} k_{j} \hat{b}_{i} \mid \Gamma_{i j}(\hat{r}+\hat{h})\right) .
\end{aligned}
$$

Using 4.7$)_{2}$ to replace $\partial_{t} \hat{b}$ and further using Cauchy-Schwarz, one has

$$
\begin{aligned}
& \partial_{t} \operatorname{Re} \sum_{i j=1}^{d}\left(\mathrm{i} k_{i} \hat{b}_{j}+\mathrm{i} k_{j} \hat{b}_{i} \mid \Gamma_{i j}(\{\mathrm{I}-\mathrm{P}\} \hat{u})+2 \hat{c} \delta_{i j}\right)+|k|^{2}|\hat{b}|^{2} \\
& \quad \leq \epsilon_{2}|k|^{2}|\hat{a}|^{2}+\frac{C}{\epsilon_{2}}|k|^{2}|\hat{c}|^{2}+\frac{C}{\epsilon_{2}}|k|^{2}\|\{\mathrm{I}-\mathrm{P}\} \hat{u}\|_{L_{\xi}^{2}}^{2}+C\left\|\nu^{-1 / 2} \hat{h}\right\|_{L_{\xi}^{2}}^{2},
\end{aligned}
$$

where $0<\epsilon_{2} \leq 1$ is arbitrary to be chosen later.

Step 3. Estimate $\hat{a}$ : Similarly, 4.7$)_{2}$ implies

$$
\begin{aligned}
|k|^{2}|\hat{a}|^{2} & =\sum_{i=1}^{d}\left(\mathrm{i} k_{i} \hat{a} \mid \mathrm{i} k_{i} \hat{a}\right)=\sum_{i=1}^{d}\left(\mathrm{i} k_{i} \hat{a} \mid-\partial_{t} \hat{b}_{i}-2 \mathrm{i} k_{i} \hat{c}-\sum_{j=1}^{d} \mathrm{i} k_{j} \Gamma_{i j}(\{\mathrm{I}-\mathrm{P}\} \hat{u})\right) \\
& =-\partial_{t} \sum_{i=1}^{d}\left(\mathrm{i} k_{i} \hat{a} \mid \hat{b}_{i}\right)+\sum_{i=1}^{d}\left(\mathrm{i} k_{i} \partial_{t} \hat{a} \mid \hat{b}_{i}\right)+\sum_{i=1}^{d}\left(\mathrm{i} k_{i} \hat{a} \mid-2 \mathrm{i} k_{i} \hat{c}-\sum_{j=1}^{d} \mathrm{i} k_{j} \Gamma_{i j}(\{\mathrm{I}-\mathrm{P}\} \hat{u})\right.
\end{aligned}
$$


which after using 4.7$]_{1}$ to replace $\partial_{t} \hat{a}$ on the r.h.s. gives

$$
\partial_{t} \operatorname{Re} \sum_{i=1}^{d}\left(\mathrm{i} k_{i} \hat{a} \mid \hat{b}_{i}\right)+\lambda|k|^{2}|\hat{a}|^{2} \leq|k \cdot \hat{b}|^{2}+C|k|^{2}|\hat{c}|^{2}+C|k|^{2}\|\{\mathrm{I}-\mathrm{P}\} \hat{u}\|_{L_{\xi}^{2}}^{2} .
$$

Finally, we define $E_{\text {int }}(\hat{u})$ by $(4.5)$. Then, for properly chosen constants $0<\kappa_{2} \ll \kappa_{1} \ll 1$ and small constants $\epsilon_{1}>0, \epsilon_{2}>0,04.6$ follows from the linear combination of the above three estimates 4.8, (4.9) and 4.10) and further dividing it by $1+|k|^{2}$. This completes the proof of Lemma 4.1 .

Remark 4.1. As in 2.6), 2.22 and (3.12), Lemma 4.1 shows that the dissipation of degenerate part which is the kernel of the linearized Boltzmann equation with dimensions equal to $d+2$ can be recovered from the fluid-type moment system (4.3). This property was firstly observed by Guo [14, 13], and it was later improved in [6, 7, 9] and [12, 18] for different purposes. Precisely, [13] is mainly based on an elliptic-type equation of b derived from [4.3 $3_{3}-(4.3)_{4}$. The aim of [6] is to remove time derivatives by constructing some functional which is similar to 4.5 but takes more complicated form. [7] introduced moment functions $\Gamma$ and $\Lambda$ to refine the form of (4.5). [9] exactly used the same method to deal with the Vlasov-Poisson-Boltzmann system. Here, it should be emphasized that due to new estimates on the dissipation of $b$, the current method of proof is more general in the sense that it can be directly modified to apply to the case with a potential forcing, see Lemma 4.3. Notice that three terms in 4.5 are inner products of $i$-th order moment and $(i+1)$-th order moment for $i=0,1,2$, respectively, and also it is in the same spirit of Kawashima's construction of compensation functions [20, 11].

Now, for $t \geq 0, k \in \mathbb{R}^{d}$, define

$$
E(\hat{u})=\|\hat{u}\|_{L_{\xi}^{2}}^{2}+\kappa_{3} \operatorname{Re} E_{\text {int }}(\hat{u})
$$

where $E_{\text {int }}(\hat{u})$ is defined by 4.5 and $\kappa_{3}>0$ is to be chosen. Similarly before, one can let $\kappa_{3}>0$ be small such that $E(\hat{u}) \sim\|\hat{u}\|_{L_{\xi}^{2}}^{2}$ and the linear combination of 4.2 and 4.6) gives

$$
\partial_{t} E(\hat{u})+\frac{\lambda|k|^{2}}{1+|k|^{2}} E(\hat{u}) \leq C\left\|\nu^{-1 / 2} \hat{h}\right\|_{L_{\xi}^{2}}^{2} .
$$

Hence, (1.14 and 1.15 follows from the above estimate in the same way as before. This completes the proof of (1.14) and (1.15) for Model 3 in Theorem 1.1

4.2 Case when $\Omega=\mathbb{T}^{d}$ and $V=0$. In this case, 1.17) follows from the same argument as for Model 1 and Model 2. In fact, it suffices to check

$$
\left.\int_{\mathbb{T}^{d}}(a, b, c) d x\right|_{t \geq 0} \equiv 0
$$

which results from the fact that it initially holds at $t=0$ by 1.16 and the conservation laws

$$
\frac{d}{d t} \int_{\mathbb{T}^{d}}(a, b, c) d x=0
$$

holds by 4.3$\left.]_{1}-4.3\right]_{3}$. This completes the proof of 1.17 in Theorem 1.1 for Model 3. 
4.3 Case when $\Omega=\mathbb{R}^{d}$ and $V$ is confining. In particular, let $V=\frac{|x|^{2}}{2}-\frac{d}{2} \ln (2 \pi)$ and $d \geq 3$. Take $u_{0}$ with

$$
\iint_{\mathbb{R}^{d} \times \mathbb{R}^{d}}\left(1, x, \xi, x \cdot \xi, x \times \xi,|x|^{2},|\xi|^{2}\right) \mathcal{M}^{1 / 2} u_{0} d x d \xi=0,
$$

where $(x \times \xi)_{i j}=x_{i} \xi_{j}-x_{j} \xi_{i}$ for $1 \leq i, j \leq d$. Let $u(t)=e^{t \mathrm{~B}} u_{0}$ be the solution to the Cauchy problem

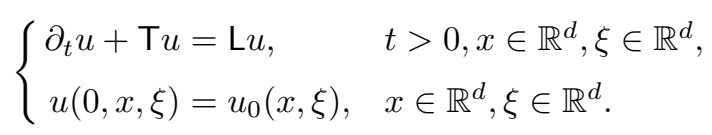

Firstly, we verify that the property (4.11) can be preserved at all time $t \geq 0$ for the above Cauchy problem 4.12 .

Lemma 4.2. Under the assumption (4.11),

$$
\iint_{\mathbb{R}^{d} \times \mathbb{R}^{d}}\left(1, x, \xi, x \cdot \xi, x \times \xi,|x|^{2},|\xi|^{2}\right) \mathcal{M}^{1 / 2} u(t, x, \xi) d x d \xi=0
$$

holds for any $t \geq 0$. Particularly, for any $t \geq 0$,

$$
\begin{gathered}
\int_{\mathbb{R}^{d}} e^{-\frac{V}{2}} a(t, x) d x=\int_{\mathbb{R}^{d}} e^{-\frac{V}{2}} b_{i}(t, x) d x=\int_{\mathbb{R}^{d}} e^{-\frac{V}{2}} c(t, x) d x=0,1 \leq i \leq d, \\
\int_{\mathbb{R}^{d}} e^{-\frac{V}{2}} x \times b(t, x) d x=0 .
\end{gathered}
$$

Proof. For simplicity, define the weighted inner product $((\cdot, \cdot))_{\mathcal{M}}$ by

$$
((u, v))_{\mathcal{M}}=\iint_{\mathbb{R}^{d} \times \mathbb{R}^{d}} u v \mathcal{M}^{1 / 2} d x d \xi .
$$

Notice that $f=\mathcal{M}+\mathcal{M}^{1 / 2} u$ satisfies 1.12 . From integration by parts, it is easy to obtain the following ODE system

$$
\left\{\begin{array}{l}
\frac{d}{d t}((1, u))_{\mathcal{M}}=0, \quad \frac{d}{d t}((x \times \xi, u))_{\mathcal{M}}=0 \\
\frac{d}{d t}((x, u))_{\mathcal{M}}=((\xi, u))_{\mathcal{M}}, \quad \frac{d}{d t}((\xi, u))_{\mathcal{M}}=-((x, u))_{\mathcal{M}} \\
\frac{d}{d t}((x \cdot \xi, u))_{\mathcal{M}}=\left(\left(|\xi|^{2}-|x|^{2}, u\right)\right)_{\mathcal{M}} \\
\frac{d}{d t}\left(\left(|x|^{2}, u\right)\right)_{\mathcal{M}}=2((x \cdot \xi, u))_{\mathcal{M}}, \quad \frac{d}{d t}\left(\left(|\xi|^{2}, u\right)\right)_{\mathcal{M}}=-2((x \cdot \xi, u))_{\mathcal{M}}
\end{array}\right.
$$

Define temporal functions

$$
y_{i}(t)=\left(\left(\xi_{i}, u\right)\right)_{\mathcal{M}} \quad(1 \leq i \leq d), \quad z(t)=((x \cdot \xi, u))_{\mathcal{M}} .
$$

Then, from (4.14) and 4.13), $y_{i}(t)$ and $z(t)$ satisfy the initial value problems of the linear second-order ODE as follows

$$
\left\{\begin{array} { l } 
{ y _ { i } ^ { \prime \prime } ( t ) + y _ { i } ( t ) = 0 , \quad t > 0 } \\
{ y _ { i } ( 0 ) = ( ( \xi , u _ { 0 } ) ) _ { \mathcal { M } } = 0 , } \\
{ y _ { i } ^ { \prime } ( 0 ) = - ( ( x _ { i } , u _ { 0 } ) ) _ { \mathcal { M } } = 0 , }
\end{array} \text { and } \quad \left\{\begin{array}{l}
z^{\prime \prime}(t)+4 z(t)=0, \quad t>0 \\
z(0)=\left(\left(x \cdot \xi, u_{0}\right)\right)_{\mathcal{M}}=0 \\
z^{\prime}(0)=-\left(\left(|\xi|^{2}-|x|^{2}, u_{0}\right)\right)_{\mathcal{M}}=0
\end{array}\right.\right.
$$

Hence, both solutions are trivial. Applying this to the system (4.14) implies that all inner products in (4.14) vanish due to 4.13). This completes the proof of Lemma 4.2 
Next, we proceed along the same line of proof as for Model 1. The zero-order energy integration of 4.121 gives

$$
\frac{1}{2} \frac{d}{d t}\|u\|^{2}+\lambda \iint_{\mathbb{R}^{d} \times \mathbb{R}^{d}} \nu(\xi)|\{\mathrm{I}-\mathrm{P}\} u|^{2} d x d \xi \leq 0 .
$$

Similar to obtain (2.14), for $\mathrm{X}$ and $\mathrm{Y}$ differentiations, one has

$$
\begin{aligned}
& \frac{1}{2} \frac{d}{d t}\left(\|\mathrm{X} u\|^{2}+\|\mathrm{Y} u\|^{2}\right)+\lambda \iint_{\mathbb{R}^{d} \times \mathbb{R}^{d}} \nu(\xi)|\{\mathrm{I}-\mathrm{P}\} \mathrm{X} u|^{2} d x d \xi \\
& \quad+\lambda \iint_{\mathbb{R}^{d} \times \mathbb{R}^{d}} \nu(\xi)|\{\mathrm{I}-\mathrm{P}\} \mathrm{Y} u|^{2} d x d \xi \leq-\sum_{i=1}^{d} \iint_{\mathbb{R}^{d} \times \mathbb{R}^{d}}\left[\mathrm{~L}, \mathrm{Y}_{i}\right] u \mathrm{Y}_{i} u d x d \xi .
\end{aligned}
$$

It is easy to check $\mathrm{Y}_{i} \mathrm{P} u \in$ ker $\mathrm{L}$ which implies

$$
\left[\mathrm{L}, \mathrm{Y}_{i}\right] u=\mathrm{LY}_{i} u-\mathrm{Y}_{i} \mathrm{~L} u=\mathrm{LY}_{i}\{\mathrm{I}-\mathrm{P}\} u-\mathrm{Y}_{i} \mathrm{~L}\{\mathrm{I}-\mathrm{P}\} u=\left[\mathrm{L}, \mathrm{Y}_{i}\right]\{\mathrm{I}-\mathrm{P}\} u \text {. }
$$

Since $\left[L, Y_{i}\right]$ is a bounded operator from $L_{\xi}^{2}$ to $L_{\xi}^{2}$ by [10],

$$
-\sum_{i=1}^{d} \iint_{\mathbb{R}^{d} \times \mathbb{R}^{d}}\left[\mathrm{~L}, \mathrm{Y}_{i}\right] u \mathrm{Y}_{i} u d x d \xi \leq \epsilon\|(a, b, c)\|^{2}+\frac{C}{\epsilon}\|\{\mathrm{I}-\mathrm{P}\} u\|^{2}
$$

for an arbitrary constant $0<\epsilon \leq 1$ to be chosen later. Then, it follows

$$
\begin{aligned}
& \frac{1}{2} \frac{d}{d t}\left(\|\mathrm{X} u\|^{2}+\|\mathrm{Y} u\|^{2}\right)+\lambda \iint_{\mathbb{R}^{d} \times \mathbb{R}^{d}} \nu(\xi)|\{\mathrm{I}-\mathrm{P}\} \mathrm{X} u|^{2} d x d \xi \\
& \quad+\lambda \iint_{\mathbb{R}^{d} \times \mathbb{R}^{d}} \nu(\xi)|\{\mathrm{I}-\mathrm{P}\} \mathrm{Y} u|^{2} d x d \xi \leq \epsilon\|(a, b, c)\|^{2}+\frac{C}{\epsilon}\|\{\mathrm{I}-\mathrm{P}\} u\|^{2} .
\end{aligned}
$$

The rest is to deal with the dissipation rate corresponding to $\mathrm{PX} u$ and $\mathrm{P} u$ or equivalently $\mathrm{X}(a, b, c)$ and $(a, b, c)$. Poincaré and Korn-type inequalities given in the next section play a key role in this step. In fact, similar to get 4.3 , from the equation $4.12{ }_{1}$ in the presence of potential forcing, one can obtain the following fluid-type system:

$$
\left\{\begin{array}{l}
\partial_{t} a-\mathbf{X}^{*} \cdot b=0 \\
\partial_{t} b+\mathrm{X} a-2 \mathbf{X}^{*} c-\mathbf{X}^{*} \cdot \Gamma(\{\mathrm{I}-\mathrm{P}\} u)=0, \\
\partial_{t} c+\frac{1}{d} \mathbf{X} \cdot b-\frac{1}{2 d} \mathbf{X}^{*} \cdot \Lambda(\{\mathrm{I}-\mathrm{P}\} u)=0 \\
\partial_{t}\left[\Gamma_{i j}(\{\mathrm{I}-\mathrm{P}\} u)+2 c \delta_{i j}\right]+\mathrm{X}_{i} b_{j}+\mathrm{X}_{j} b_{i}=\Gamma_{i j}(r), \\
\partial_{t} \Lambda_{i}(\{\mathrm{I}-\mathrm{P}\} u)+\mathbf{X}_{i} c=\Lambda_{i}(r),
\end{array}\right.
$$

where moment functions $\Gamma$ and $\Lambda$ are defined as in (2.5) and (4.4) respectively, and for simplicity, we still used $r$ to denote

$$
r=-\xi \cdot \nabla_{x}\{\mathrm{I}-\mathrm{P}\} u+\nabla_{x} V \cdot \nabla_{\xi}\{\mathrm{I}-\mathrm{P}\} u+\mathrm{L}\{\mathrm{I}-\mathrm{P}\} u .
$$

Lemma 4.3. There exists a temporal functional $\mathcal{E}_{\text {int }}(u(t))$ given by

$$
\begin{aligned}
\mathcal{E}_{\text {int }}(u(t))= & \sum_{i=1}^{d}\left(\mathrm{X}_{i} c, \Lambda_{i}(\{\mathrm{I}-\mathrm{P}\} u)\right) \\
& +\kappa_{1} \sum_{i j=1}^{d}\left(\mathrm{X}_{i} b_{j}+\mathrm{X}_{j} b_{i}, \Gamma_{i j}(\{\mathrm{I}-\mathrm{P}\} u)+2 c \delta_{i j}\right)+\kappa_{2} \sum_{i=1}^{d}\left(\mathrm{X}_{i} a, b_{i}\right)
\end{aligned}
$$


for constants $0<\kappa_{2} \ll \kappa_{1} \ll 1$ such that there are constants $\lambda>0$, C such that

$$
\frac{d}{d t} \mathcal{E}_{\text {int }}(u(t))+\lambda \mathcal{D}(a, b, c) \leq C\left(\|\{\mathrm{I}-\mathrm{P}\} \mathrm{X} u\|^{2}+\|\{\mathrm{I}-\mathrm{P}\} u\|^{2}\right)
$$

holds for any $t \geq 0$, where the dissipation rate $\mathcal{D}(a, b, c)$ denotes

$$
\mathcal{D}(a, b, c)=\left(\|\mathrm{X} a\|^{2}+\|a\|^{2}\right)+\sum_{i=1}^{d}\left(\left\|\mathbf{X} b_{i}\right\|^{2}+\left\|b_{i}\right\|^{2}\right)+\left(\|\mathrm{X} c\|^{2}+\|c\|^{2}\right)
$$

Proof. This follows from the same procedure as for the proof of Lemma 4.1 which is on the basis of Fourier transform when there is no external forcing. For completeness, we shall provide all details of proof. Firstly, it is straightforward to check

$$
\begin{aligned}
\|\Gamma(r)\|^{2}+\|\Lambda(r)\|^{2} & \leq C \iint_{\mathbb{R}^{d} \times \mathbb{R}^{d}}\left|\nabla_{x}\{\mathrm{I}-\mathrm{P}\} u\right|^{2}+|\{\mathrm{I}-\mathrm{P}\} u|^{2}\left(1+\left|\nabla_{x} V\right|^{2}\right) d x d \xi \\
& \leq C\left(\|\{\mathrm{I}-\mathrm{P}\} \mathrm{X} u\|^{2}+\|\{\mathrm{I}-\mathrm{P}\} u\|^{2}\right) .
\end{aligned}
$$

Step 1. Estimate on $c: 4.17)_{5}$ gives

$$
\begin{aligned}
\|\mathrm{X} c\|^{2} & =\sum_{i=1}^{d}\left(\mathrm{X}_{i} c,-\partial_{t} \Lambda_{i}(\{\mathrm{I}-\mathrm{P}\} u)+\Lambda_{i}(r)\right) \\
& =-\frac{d}{d t} \sum_{i=1}^{d}\left(\mathrm{X}_{i} c, \Lambda_{i}(\{\mathrm{I}-\mathrm{P}\} u)\right)+\sum_{i=1}^{d}\left(\mathrm{X}_{i} \partial_{t} c, \Lambda_{i}(\{\mathrm{I}-\mathrm{P}\} u)\right)+\sum_{i=1}^{d}\left(\mathrm{X}_{i} c, \Lambda_{i}(r)\right) .
\end{aligned}
$$

Using 4.17$]_{3}$ to replace $\partial_{t} c$, one has

$$
\begin{aligned}
\sum_{i=1}^{d}\left(\mathrm{X}_{i} \partial_{t} c, \Lambda_{i}(\{\mathrm{I}-\mathrm{P}\} u)\right. & =\left(-\frac{1}{d} \mathrm{X} \cdot b+\frac{1}{2 d} \mathrm{X}^{*} \cdot \Lambda(\{\mathrm{I}-\mathrm{P}\} u), \mathrm{X}^{*} \cdot \Lambda(\{\mathrm{I}-\mathrm{P}\} u)\right) \\
& \leq \epsilon_{1}\|\mathrm{X} \cdot b\|^{2}+\frac{C}{\epsilon_{1}}\left\|\mathrm{X}^{*}\{\mathrm{I}-\mathrm{P}\} u\right\|^{2}
\end{aligned}
$$

for an arbitrary constant $0<\epsilon_{1} \leq 1$ to be chosen later. In addition, it holds

$$
\sum_{i=1}^{d}\left(\mathrm{X}_{i} c, \Lambda_{i}(r)\right) \leq \frac{1}{2}\|\mathrm{X} c\|^{2}+C\|\Lambda(r)\|^{2}
$$

By putting the above two estimates into 4.21 and using 4.20 and Lemma 2.1. one has

$$
\begin{aligned}
\frac{d}{d t} \sum_{i=1}^{d}\left(\mathrm{X}_{i} c, \Lambda_{i}(\{\mathrm{I}-\mathrm{P}\} u)\right)+\lambda\left(\|\mathrm{X} c\|^{2}+\|c\|^{2}\right) \\
\leq \epsilon_{1}\|\mathrm{X} \cdot b\|^{2}+\frac{C}{\epsilon_{1}}\left(\|\{\mathrm{I}-\mathrm{P}\} \mathrm{X} u\|^{2}+\|\{\mathrm{I}-\mathrm{P}\} u\|^{2}\right)
\end{aligned}
$$

where due to Lemma 4.2 and Poincaré inequality in Proposition 5.1 , $\|c\|^{2}$ was included in the dissipation rate. 
Step 2. Estimate on $b: 4.17)_{4}$ gives

$$
\begin{aligned}
\sum_{i j=1}^{d}\left\|\mathrm{X}_{i} b_{j}+\mathrm{X}_{j} b_{i}\right\|^{2}= & -\frac{d}{d t} \sum_{i j=1}^{d}\left(\mathrm{X}_{i} b_{j}+\mathrm{X}_{j} b_{i}, \Gamma_{i j}(\{\mathrm{I}-\mathrm{P}\} u)+2 c \delta_{i j}\right) \\
& +\sum_{i j=1}^{d}\left(\mathrm{X}_{i} \partial_{t} b_{j}+\mathrm{X}_{j} \partial_{t} b_{i}, \Gamma_{i j}(\{\mathrm{I}-\mathrm{P}\} u)+2 c \delta_{i j}\right) \\
& +\sum_{i j=1}^{d}\left(\mathrm{X}_{i} b_{j}+\mathrm{X}_{j} b_{i}, \Gamma_{i j}(r)\right)
\end{aligned}
$$

From 4.17$)_{2}, \partial_{t} b$ is replaced to obtain

$$
\begin{aligned}
& \sum_{i j=1}^{d}\left(\mathrm{X}_{i} \partial_{t} b_{j}+\mathrm{X}_{j} \partial_{t} b_{i}, \Gamma_{i j}(\{\mathrm{I}-\mathrm{P}\} u)+2 c \delta_{i j}\right) \\
& =2 \sum_{i j=1}^{d}\left(\partial_{t} b_{i}, \mathrm{X}_{j}^{*} \Gamma_{i j}(\{\mathrm{I}-\mathrm{P}\} u)+2 \mathbf{X}_{j}^{*} c \delta_{i j}\right) \\
& =2 \sum_{i j=1}^{d}\left(-\mathbf{X}_{i} a+2 \mathbf{X}_{i}^{*} c+\sum_{\ell=1}^{d} \mathbf{X}_{\ell}^{*} \Gamma_{\ell i}(\{\mathrm{I}-\mathrm{P}\} u), \mathrm{X}_{j}^{*} \Gamma_{i j}(\{\mathrm{I}-\mathrm{P}\} u)+2 \mathbf{X}_{j}^{*} c \delta_{i j}\right) \\
& \leq \epsilon_{2}\|\mathbf{X} a\|^{2}+\frac{C}{\epsilon_{2}}\left(\left\|\mathbf{X}^{*} c\right\|^{2}+\|\mathbf{X} c\|^{2}\right)+\frac{C}{\epsilon_{2}}\left\|\mathbf{X}^{*}\{\mathbf{I}-\mathrm{P}\} u\right\|^{2}
\end{aligned}
$$

for an arbitrary constant $0<\epsilon_{2} \leq 1$ to be chosen later. The final term on the r.h.s. of 4.23) is bounded by

$$
\sum_{i j=1}^{d}\left(\mathrm{X}_{i} b_{j}+\mathrm{X}_{j} b_{i}, \Gamma_{i j}(r)\right) \leq \frac{1}{2} \sum_{i j=1}^{d}\left\|\mathrm{X}_{i} b_{j}+\mathrm{X}_{j} b_{i}\right\|^{2}+C\|\Gamma(r)\|^{2} .
$$

Similar to Step 1, it follows from 4.23 that

$$
\begin{aligned}
& \frac{d}{d t} \sum_{i j=1}^{d}\left(\mathrm{X}_{i} b_{j}+\mathrm{X}_{j} b_{i}, \Gamma_{i j}(\{\mathrm{I}-\mathrm{P}\} u)+2 c \delta_{i j}\right)+\lambda \sum_{i=1}^{d}\left(\left\|\mathrm{X} b_{i}\right\|^{2}+\left\|b_{i}\right\|^{2}\right) \\
& \quad \leq \epsilon_{2}\|\mathrm{X} a\|^{2}+\frac{C}{\epsilon_{2}}\left(\|\mathrm{X} c\|^{2}+\|c\|^{2}\right)+\frac{C}{\epsilon_{2}}\left(\|\{\mathrm{I}-\mathrm{P}\} \mathrm{X} u\|^{2}+\|\{\mathrm{I}-\mathrm{P}\} u\|^{2}\right),
\end{aligned}
$$

where due to Lemma 4.2 Korn inequalities (5.5) and (5.14) were used.

Step 3. Estimate on $a: 4.17)_{2}$ implies

$$
\|\mathrm{X} a\|^{2}=-\frac{d}{d t} \sum_{i=1}^{d}\left(\mathrm{X}_{i} a, b_{i}\right)+\sum_{i=1}^{d}\left(\mathrm{X}_{i} \partial_{t} a, b_{i}\right)+\sum_{i=1}^{d}\left(\mathrm{X}_{i} a, 2 \mathbf{X}_{i}^{*} c+\sum_{j=1}^{d} \mathrm{X}_{j}^{*} \Gamma_{i j}(\{\mathbf{I}-\mathrm{P}\} u)\right),
$$

where it further holds that

$$
\sum_{i=1}^{d}\left(\mathrm{X}_{i} \partial_{t} a, b_{i}\right)=\left(\partial_{t} a, \mathrm{X}^{*} \cdot b\right)=\left\|\mathrm{X}^{*} \cdot b\right\|^{2} \leq C \sum_{i=1}^{d}\left(\left\|\mathrm{X} b_{i}\right\|^{2}+\left\|b_{i}\right\|^{2}\right)
$$


by 4.17 , and

$$
\sum_{i=1}^{d}\left(\mathrm{X}_{i} a, 2 \mathbf{X}_{i}^{*} c+\sum_{j=1}^{d} \mathrm{X}_{j}^{*} \Gamma_{i j}(\{\mathrm{I}-\mathrm{P}\} u)\right) \leq \frac{1}{2}\|\mathrm{X} a\|^{2}+C\left\|\mathbf{X}^{*} c\right\|^{2}+C\left\|\mathbf{X}^{*}\{\mathrm{I}-\mathrm{P}\} u\right\|^{2} .
$$

Then, it follows

$$
\begin{gathered}
\frac{d}{d t} \sum_{i=1}^{d}\left(\mathrm{X}_{i} a, b_{i}\right)+\lambda\left(\|\mathrm{X} a\|^{2}+\|a\|^{2}\right) \\
\leq C \sum_{i=1}^{d}\left(\left\|\mathrm{X} b_{i}\right\|^{2}+\left\|b_{i}\right\|^{2}\right)+C\left(\|\mathrm{X} c\|^{2}+\|c\|^{2}\right)+C\left(\|\{\mathrm{I}-\mathrm{P}\} \mathrm{X} u\|^{2}+\|\{\mathrm{I}-\mathrm{P}\} u\|^{2}\right),
\end{gathered}
$$

where again due to Lemma 4.2. Poincaré inequality in Proposition 5.1 was used for $a$.

Finally, define $\mathcal{E}_{\text {int }}(u(t))$ as in 4.18). Then, for properly chosen constants $0<\kappa_{2} \ll$ $\kappa_{1} \ll 1$ and small constants $\epsilon_{1}>0, \epsilon_{2}>0,(4.19)$ follows from the linear combination of three inequalities 4.22, (4.24) and 4.25). This completes the proof of Lemma 4.3 .

Now, for $t \geq 0$, define

$$
\mathcal{E}(u(t))=\|u\|^{2}+\kappa_{3}\left(\|\mathrm{X} u\|^{2}+\|\mathrm{Y} u\|^{2}\right)+\kappa_{4} \mathcal{E}_{\text {int }}(u(t))
$$

where $\mathcal{E}_{\text {int }}(u(t))$ is defined by 4.18 and constants $0<\kappa_{4} \ll \kappa_{3} \ll 1$ are to be chosen later. By letting $0<\kappa_{4} \ll \kappa_{3} \ll 1$ be properly chosen, one has $\mathcal{E}(u(t)) \sim\|u(t)\|_{\mathcal{H}^{1}}^{2}$. On the other hand, by further properly choosing $0<\kappa_{4} \ll \kappa_{3} \ll 1$, the linear combination of 4.15, 4.16) and 4.19 yields

$$
\frac{d}{d t} \mathcal{E}(u(t))+\lambda \mathcal{E}(u(t)) \leq 0,
$$

where $\epsilon>0$ in 4.16 was also chosen small enough. Hence, 1.18 follows from Gronwall inequality. This completes the proof of Theorem 1.2 for Model 3.

\section{Appendix: Poincaré and Korn-type inequalities}

Let $V=V(x)$ be a smooth confining function over $\mathbb{R}^{d}$ with

$$
\int_{\mathbb{R}^{d}} e^{-V(x)} d x=1
$$

We need assumptions on $V$ :

$$
\frac{1}{4}|\nabla V|^{2}-\frac{1}{2} \Delta V \rightarrow \infty \text { as }|x| \rightarrow \infty
$$

or

$$
\frac{1}{4}|\nabla V|^{2}-\frac{1}{2} \Delta V-\sum_{i j=1}^{d}\left|\partial_{i} \partial_{j} V\right| \rightarrow \infty \quad \text { as }|x| \rightarrow \infty .
$$

Define operators $\mathrm{X}_{i}(1 \leq i \leq d)$ associated with $V$ as in 1.3 . Throughout this section, differential operators $\nabla, \Delta, \nabla^{2}$ denotes $\nabla_{x}, \Delta_{x}, \nabla_{x}^{2}$ for simplicity since all functions are those of spatial variable. The following Poincaré inequality in $\mathbb{R}^{d}$ has been used in this paper. 
Proposition 5.1. Let $d \geq 1$. Suppose 5.1 and 5.2 hold. There is a constant $\lambda>0$ such that

$$
\sum_{i=1}^{d} \int_{\mathbb{R}^{d}}\left|\mathbf{X}_{i} a\right|^{2} d x \geq \lambda \int_{\mathbb{R}^{d}}|a|^{2} d x
$$

holds for any $a: \mathbb{R}^{d} \rightarrow \mathbb{R} \in L^{2}\left(\mathbb{R}^{d}\right)$ satisfying

$$
\int_{\mathbb{R}^{d}} e^{-\frac{V(x)}{2}} a d x=0
$$

For the proof of the above proposition, refer to [28] on the basis of the constructive method, where $\lambda$ in (5.4) can be explicitly computed.

The following Korn-type inequality in $\mathbb{R}^{d}$ was also essentially used in the previous proof to obtain the dissipation of the macroscopic momentum. For the study of the Korn inequality in other situations, refer to [3] and reference therein.

Theorem 5.1. Let $d \geq 2$. Suppose (5.1) and 5.3) hold. There is a constant $\lambda>0$ such that

$$
\sum_{i j=1}^{d} \int_{\mathbb{R}^{d}}\left|\mathrm{X}_{i} b_{j}+\mathrm{X}_{j} b_{i}\right|^{2} d x \geq \lambda \sum_{i=1}^{d} \int_{\mathbb{R}^{d}}\left|b_{i}\right|^{2} d x
$$

holds for any $b=\left(b_{1}, b_{2}, \cdots, b_{d}\right): \mathbb{R}^{d} \rightarrow \mathbb{R}^{d} \in\left(L^{2}\left(\mathbb{R}^{d}\right)\right)^{d}$ satisfying

$$
\int_{\mathbb{R}^{d}} e^{-\frac{V(x)}{2}} b(x) d x=0
$$

and

$$
\int_{\mathbb{R}^{d}} e^{-\frac{V(x)}{2}}\left(x_{i} b_{j}-x_{j} b_{i}\right) d x=0, \quad 1 \leq i \neq j \leq d .
$$

Proof. We use the contradiction argument.

Step 1. Otherwise, for any $n \geq 1$, there is a nonzero element $b^{n}=\left(b_{1}^{n}, b_{2}^{n}, \cdots, b_{d}^{n}\right) \in\left(L^{2}\left(\mathbb{R}^{d}\right)\right)^{d}$ with

$$
\int_{\mathbb{R}^{d}} e^{-\frac{V(x)}{2}} b^{n}(x) d x=0, \quad \int_{\mathbb{R}^{d}} e^{-\frac{V(x)}{2}} x \times b^{n}(x) d x=0,
$$

such that

$$
\sum_{i j=1}^{d} \int_{\mathbb{R}^{d}}\left|\mathrm{X}_{i} b_{j}^{n}+\mathrm{X}_{j} b_{i}^{n}\right|^{2} d x \leq \frac{1}{n} \sum_{i=1}^{d} \int_{\mathbb{R}^{d}}\left|b_{i}^{n}\right|^{2} d x .
$$

W.l.g., one can suppose $\sum_{i=1}^{d} \int_{\mathbb{R}^{d}}\left|b_{i}^{n}\right|^{2} d x=1$. Thus,

$$
\sum_{i j=1}^{d} \int_{\mathbb{R}^{d}}\left|\mathbf{X}_{i} b_{j}^{n}+\mathbf{X}_{j} b_{i}^{n}\right|^{2} d x \leq \frac{1}{n} .
$$

Step 2. We claim that there is a constant $C$ independent of $n \geq 1$ and a continuous function $w(x)$ only depending on $V$ with $\inf _{x \in \mathbb{R}^{d}} w(x) \geq 1$ and $w(x) \rightarrow \infty$ as $|x| \rightarrow \infty$, such that

$$
\sup _{n \geq 1} \int_{\mathbb{R}^{d}}\left|\nabla b^{n}\right|^{2}+\left|b^{n}\right|^{2} w(x) d x \leq C .
$$


In fact, one can compute

$$
\sum_{i j=1}^{d} \int_{\mathbb{R}^{d}}\left|\mathbf{X}_{i} b_{j}^{n}+\mathbf{X}_{j} b_{i}^{n}\right|^{2} d x=2 \sum_{i j=1}^{d} \int_{\mathbb{R}^{d}}\left|\mathbf{X}_{i} b_{j}^{n}\right|^{2} d x+2 \sum_{i j=1}^{d}\left(\mathbf{X}_{i} b_{j}^{n}, \mathbf{X}_{j} b_{i}^{n}\right):=I_{1}+I_{2} .
$$

The tedious computations from integration by parts show

$$
\begin{aligned}
I_{1}+I_{2}= & 2 \int_{\mathbb{R}^{d}}\left|\nabla b^{n}\right|^{2}+\left|b^{n}\right|^{2}\left(\frac{1}{4}|\nabla V|^{2}-\frac{1}{2} \Delta V\right) d x \\
& +\left\|\frac{1}{2} \nabla V \cdot b^{n}-\nabla \cdot b^{n}\right\|^{2}-2\left(b^{n}, \nabla^{2} V b^{n}\right) .
\end{aligned}
$$

Then, this implies

$$
\begin{aligned}
I_{1}+I_{2} \geq & 2 \int_{\mathbb{R}^{d}}\left|\nabla b^{n}\right|^{2}+\left|b^{n}\right|^{2}\left(\frac{1}{4}|\nabla V|^{2}-\frac{1}{2} \Delta V-\sum_{i j=1}^{d}\left|\partial_{i} \partial_{j} V\right|\right) d x \\
& +\left\|\frac{1}{2} \nabla V \cdot b^{n}-\nabla \cdot b^{n}\right\|^{2} .
\end{aligned}
$$

Now, define $w(x)=\max \left\{\frac{1}{4}|\nabla V|^{2}-\frac{1}{2} \Delta V-\sum_{i j=1}^{d}\left|\partial_{i} \partial_{j} V\right|, 1\right\}$. Due to (5.3), $w(x) \rightarrow \infty$ as $|x| \rightarrow \infty$. Choosing $R>0$ large enough, one has $w(x)=\frac{1}{4}|\nabla V|^{2}-\frac{1}{2} \Delta V-\sum_{i j=1}^{d}\left|\partial_{i} \partial_{j} V\right|$ for $|x| \geq R$. Then

$$
\int_{\mathbb{R}^{d}}\left|b^{n}\right|^{2}\left(\frac{1}{4}|\nabla V|^{2}-\frac{1}{2} \Delta V-\sum_{i j=1}^{d}\left|\partial_{i} \partial_{j} V\right|\right) d x \geq \int_{\mathbb{R}^{d}}\left|b^{n}\right|^{2} w(x) d x-C_{R, V}
$$

where

$$
C_{R, V}=\sup _{|x| \leq R} w(x)-\inf _{|x| \leq R}\left(\frac{1}{4}|\nabla V|^{2}-\frac{1}{2} \Delta V-\sum_{i j=1}^{d}\left|\partial_{i} \partial_{j} V\right|\right) .
$$

Then, it follows that

$$
\begin{gathered}
\sum_{i j=1}^{d} \int_{\mathbb{R}^{d}}\left|\mathrm{X}_{i} b_{j}^{n}+\mathrm{X}_{j} b_{i}^{n}\right|^{2} d x=I_{1}+I_{2} \\
\geq 2 \int_{\mathbb{R}^{d}}\left|\nabla b^{n}\right|^{2}+\left|b^{n}\right|^{2} w(x) d x+\left\|\frac{1}{2} \nabla V \cdot b^{n}-\nabla \cdot b^{n}\right\|^{2}-2 C_{R, V},
\end{gathered}
$$

which implies that for all $n \geq 1$,

$$
\int_{\mathbb{R}^{d}}\left|\nabla b^{n}\right|^{2}+\left|b^{n}\right|^{2} w(x) d x \leq \frac{1}{2} \sum_{i j=1}^{d} \int_{\mathbb{R}^{d}}\left|\mathbf{X}_{i} b_{j}^{n}+\mathbf{X}_{j} b_{i}^{n}\right|^{2} d x+C_{R, V} \leq \frac{1}{2}+C_{R, V}
$$

Hence, the claim follows.

Step 3. From the Rellich-Kondrachov compactness theorem, up to a subsequence, $\left(b^{n}\right)_{n \geq 1}$ strongly converges to some function $b \in\left(L^{2}\left(\mathbb{R}^{d}\right)\right)^{d}$, i.e. $\left\|b^{n}-b\right\| \rightarrow 0$ as $n \rightarrow \infty$. Thus, $b$ satisfies

$$
\|b\|=1
$$


and

$$
\begin{gathered}
X_{i} b_{j}+X_{j} b_{i}=0, \quad 1 \leq i, j \leq d, \\
\int_{\mathbb{R}^{d}} e^{-\frac{V(x)}{2}} b(x) d x=0, \\
\int_{\mathbb{R}^{d}} e^{-\frac{V(x)}{2}}\left(x_{i} b_{j}-x_{j} b_{i}\right) d x=0, \quad 1 \leq i \neq j \leq d .
\end{gathered}
$$

Step 4. We claim that (5.9), (5.10) and (5.11) imply $b=0$, which is a contradiction to (5.8). In fact, set $b_{i}(x)=e^{-\frac{V}{2}} \tilde{b}_{i}(x), i=1,2, \cdots, d$. From $X_{i} b_{i}=0, \partial_{i} \tilde{b}_{i}=0$. Then, $\tilde{b}_{i}(x)$ is independent of $x_{i}$. Thus, set $\tilde{b}_{i}=\tilde{b}_{i}\left(\tilde{x}_{i}\right)$, where $\tilde{x}_{i}$ is a variable $\left(x_{1}, x_{2}, \cdots, x_{d}\right)$ excluding $x_{i}$. From (5.9) for $i \neq j$, one has

$$
\partial_{i} \tilde{b}_{j}\left(\tilde{x}_{j}\right)+\partial_{j} \tilde{b}_{i}\left(\tilde{x}_{i}\right)=0, \quad i \neq j .
$$

This implies that $\tilde{b}_{i}\left(\tilde{x}_{i}\right)$ is linear in each $x_{j}(j \neq i)$. Thus,

$$
\tilde{b}_{i}\left(\tilde{x}_{i}\right)=\sum_{\left\{\alpha: \alpha_{i}=0, \alpha_{j}=0 \text { or } 1, j \neq i\right\}} C_{\alpha}^{i} x^{\alpha} .
$$

It suffices to prove all coefficients $C_{\alpha}^{i}$ vanish. In what follows, fix $i \in\{1, \cdots, d\}$.

Case 1. $|\alpha|=0$. Due to 5.10), $C_{\alpha}^{i}=0$.

Case 2. $2 \leq|\alpha| \leq d-1$. Let $\ell=|\alpha|$ and take $\left\{i_{1}, i_{2}, \cdots, i_{\ell}\right\} \subset\{1,2, \cdots, d\}$. Consider the coefficient of the monomial $\Pi_{j=1}^{k} x_{i_{j}}$. It only appears in $\tilde{b}_{j}$ with $j \in\{1,2, \cdots, d\} \backslash\left\{i_{1}, i_{2}, \cdots, i_{\ell}\right\}$. Take $i_{\ell+1} \in\{1,2, \cdots, d\} \backslash\left\{i_{1}, i_{2}, \cdots, i_{\ell}\right\}$. Consider the following three functions

$$
\begin{aligned}
\tilde{b}_{i_{\ell+1}} & =\cdots+C_{1} x_{i_{1}} x_{i_{2}} \cdots x_{i_{\ell-1}} x_{i_{\ell}}+\cdots, \\
\tilde{b}_{i_{\ell}} & =\cdots+C_{2} x_{i_{1}} x_{i_{2}} \cdots x_{i_{\ell-1}} x_{i_{\ell+1}}+\cdots, \\
\tilde{b}_{i_{\ell-1}} & =\cdots+C_{3} x_{i_{1}} x_{i_{2}} \cdots x_{i_{\ell-2}} x_{i_{\ell}} x_{i_{\ell+1}}+\cdots,
\end{aligned}
$$

where $C_{1}, C_{2}, C_{3}$ are the corresponding constant coefficients. Due to 5.12 , one has $C_{1}+C_{2}=0$, $C_{2}+C_{3}=0$, and $C_{3}+C_{1}=0$. Thus, $C_{1}=C_{2}=C_{3}=0$. The coefficient of $\Pi_{j=1}^{k} x_{i_{j}}$ is zero. This proves $C_{\alpha}^{i}=0$ when $2 \leq|\alpha| \leq d-1$.

Case 3. $|\alpha|=1$. Take $j \neq i$. Consider the function

$$
\tilde{b}_{j}=\cdots+C_{i} x_{i}+\cdots,
$$

where $C_{i}$ is a constant. Due to $5.12, \tilde{b}_{i}$ is in the form of

$$
\tilde{b}_{i}=\cdots-C_{i} x_{j}+\cdots .
$$

By using (5.11), one has

$$
0=\int_{\mathbb{R}^{d}} e^{-V}\left(x_{i} \tilde{b}_{j}-x_{j} \tilde{b}_{i}\right) d x=C_{i} \int_{\mathbb{R}^{d}} e^{-V}\left(x_{i}^{2}+x_{j}^{2}\right) d x .
$$

Then, $C_{i}=0$. Thus, $C_{\alpha}^{i}=0$ when $|\alpha|=1$. This also completes the proof of Theorem 5.1 . 
Corollary 5.1. Let $d \geq 2$. Suppose (5.1) and 5.3 hold. Further assume that there is a constant $C$ such that

$$
\left|\nabla^{2} V\right|^{2} \leq C\left(|\nabla V|^{2}+1\right)
$$

for all $x \in \mathbb{R}^{d}$. Then, there is a constant $\lambda>0$ such that

$$
\sum_{i j=1}^{d} \int_{\mathbb{R}^{d}}\left|\mathbf{X}_{i} b_{j}+\mathbf{X}_{j} b_{i}\right|^{2} d x \geq \lambda \sum_{i j=1}^{d} \int_{\mathbb{R}^{d}}\left|\mathbf{X}_{i} b_{j}\right|^{2} d x
$$

holds for any $b=\left(b_{1}, b_{2}, \cdots, b_{d}\right): \mathbb{R}^{d} \rightarrow \mathbb{R}^{d} \in\left(L^{2}\left(\mathbb{R}^{d}\right)\right)^{d}$ satisfying

$$
\int_{\mathbb{R}^{d}} e^{-\frac{V(x)}{2}} b(x) d x=0
$$

and

$$
\int_{\mathbb{R}^{d}} e^{-\frac{V(x)}{2}}\left(x_{i} b_{j}-x_{j} b_{i}\right) d x=0, \quad 1 \leq i \neq j \leq d .
$$

Proof. Recall from 5.6 and 5.7 that one has the inequality

$$
\begin{gathered}
\sum_{i j=1}^{d} \int_{\mathbb{R}^{d}}\left|\mathrm{X}_{i} b_{j}+\mathrm{X}_{j} b_{i}\right|^{2} d x \\
\geq 2 \int_{\mathbb{R}^{d}}|\nabla b|^{2}+|b|^{2}\left(\frac{1}{4}|\nabla V|^{2}-\frac{1}{2} \Delta V-\sum_{i j=1}^{d}\left|\partial_{i} \partial_{j} V\right|\right) d x .
\end{gathered}
$$

From the assumption 5.13 , one can show that

$$
|\nabla V|^{2} \geq \lambda\left(\frac{1}{4}|\nabla V|^{2}-\frac{1}{2} \Delta V\right)-C_{\lambda}
$$

for some constant $0<\lambda<4$, and

$$
\frac{1}{4}|\nabla V|^{2}-\frac{1}{2} \Delta V-\sum_{i j=1}^{d}\left|\partial_{i} \partial_{j} V\right| \geq\left(\frac{1}{4}-\epsilon\right)|\nabla V|^{2}-C_{\epsilon}
$$

for any $\epsilon>0$. Then, combining (5.16) and (5.17), it follows

$$
\frac{1}{4}|\nabla V|^{2}-\frac{1}{2} \Delta V-\sum_{i j=1}^{d}\left|\partial_{i} \partial_{j} V\right| \geq \lambda\left(\frac{1}{4}|\nabla V|^{2}-\frac{1}{2} \Delta V\right)-C_{\lambda}
$$

for some constant $0<\lambda<1$. Plugging the above inequality into 5.15 gives

$$
\sum_{i j=1}^{d} \int_{\mathbb{R}^{d}}\left|\mathbf{X}_{i} b_{j}+\mathbf{X}_{j} b_{i}\right|^{2} d x \geq \lambda \sum_{i j=1}^{d} \int_{\mathbb{R}^{d}}\left|\mathbf{X}_{i} b_{j}\right|^{2} d x-C_{\lambda} \int_{\mathbb{R}^{d}}|b|^{2} d x
$$

This together with the Korn-type inequality (5.5) prove (5.14) and thus complete the proof of Corollary 5.1 . 
We conclude this paper with a remark that Proposition 5.1. Theorem 5.1 and Corollary 5.1 can be described in terms of the probability measure $d \mu=e^{-V(x)} d x$ as in [28, where $V$ satisfies the corresponding conditions. In fact, equivalently, (5.4 become

$$
\int_{\mathbb{R}^{d}}|\nabla a|^{2} d \mu \geq \lambda \int_{\mathbb{R}^{d}}|a|^{2} d \mu
$$

for $a$ with $\int_{\mathbb{R}^{d}} a d \mu=0$. Similarly, 5.5 and 5.14 are respectively equivalent with

$$
\sum_{i j=1}^{d} \int_{\mathbb{R}^{d}}\left|\partial_{i} b_{j}+\partial_{j} b_{i}\right|^{2} d \mu \geq \lambda \sum_{i=1}^{d} \int_{\mathbb{R}^{d}}\left|b_{i}\right|^{2} d \mu
$$

and

$$
\sum_{i j=1}^{d} \int_{\mathbb{R}^{d}}\left|\partial_{i} b_{j}+\partial_{j} b_{i}\right|^{2} d \mu \geq \lambda \sum_{i j=1}^{d} \int_{\mathbb{R}^{d}}\left|\partial_{i} b_{j}\right|^{2} d \mu
$$

for $b=\left(b_{1}, b_{2}, \cdots, b_{d}\right)$ with $\int_{\mathbb{R}^{d}} b d \mu=0$ and $\int_{\mathbb{R}^{d}} x \times b d \mu=0$.

Acknowledgments: This project was partially supported by the Direct Grant 2010/2011. The author would like to thank Jean Dolbeault, Clément Mouhot and Frédéric Hérau for their helpful discussions and suggestions for the development of this work, and also thank Peter Markowich and Massimo Fornasier for their strong support during his study at RICAM in year 2009. The author also thanks the anonymous referee for useful comments.

\section{References}

[1] M.-J. Cáceres, J.-A. Carrillo and T. Goudon, Equilibration rate for the linear inhomogeneous relaxation-time Boltzmann equation for charged particles, Comm. Partial Differential Equations 28 (2003), 969-989.

[2] C. Cercignani, R. Illner and M. Pulvirenti, The Mathematical Theory of Dilute Gases, Applied Mathematical Sciences, 106. Springer-Verlag, New York, 1994.

[3] L. Desvillettes and C. Villani, On a variant of Korn's inequality arising in statistical mechanics, ESAIM Control Optim. Calc. Var. 8 (2002), 603-619.

[4] L. Desvillettes and C. Villani, On the trend to global equilibrium for spatially inhomogeneous kinetic systems: The Boltzmann equation. Invent. Math. 159 (2) (2005), 245-316.

[5] J. Dolbeault, C. Mouhot and C. Schmeiser, Hypocoercivity for kinetic equations with linear relaxation terms, C.R. Acad. Sci. Paris 347 (2009), 511-516.

[6] R.-J. Duan, On the Cauchy problem for the Boltzmann equation in the whole space: Global existence and uniform stability in $L_{\xi}^{2}\left(H_{x}^{N}\right)$, Journal of Differential Equations 244 (2008), 3204-3234.

[7] R.-J. Duan, Stability of the Boltzmann equation with potential forces on Torus, Physica D: Nonlinear Phenomena 238 (2009), 1808-1820.

[8] R.-J. Duan, M. Fornasier and G. Toscani, A kinetic flocking model with diffusions, Communications in Mathematical Physics 300(1)(2010), 95-145.

[9] R.-J. Duan and R.M. Strain, Optimal time decay of the Vlasov-Poisson-Boltzmann system in $\mathbb{R}^{3}$, Archive for Rational Mechanics and Analysis 199(1)(2011), 291-328. 
Hypocoercivity of Linear Degenerately Dissipative Kinetic Equations

[10] R.-J. Duan, S. Ukai, T. Yang and H.-J. Zhao, Optimal decay estimates on the linearized Boltzmann equation with time-dependent forces and their applications, Communications in Mathematical Physics 277 (1)(2008), 189-236.

[11] R.T. Glassey, The Cauchy Problem in Kinetic Theory. Society for Industrial and Applied Mathematics (SIAM), Philadelphia, PA, 1996. xii+241 pp.

[12] Y. Guo, Boltzmann diffusive limit beyond the Navier-Stokes approximation, Comm. Pure Appl. Math. 59 (2006), 626-687.

[13] Y. Guo, The Boltzmann equation in the whole space, Indiana Univ. Math. J. 53 (2004), 1081-1094.

[14] Y. Guo, The Landau equation in a periodic box, Commun. Math. Phys. 231 (2002), 391434.

[15] F. Hérau, Hypocoercivity and exponential time decay for the linear inhomogeneous relaxation Boltzmann equation, Asymptot. Anal. 46 (2006), 349-359.

[16] F. Hérau and F. Nier, Isotropic hypoellipticity and trend to equilibrium for the FokkerPlanck equation with a high-degree potential, Arch. Ration. Mech. Anal. 171 (2004), $151-218$.

[17] F. Hérau, Short and long time behavior of the Fokker-Planck equation in a confining potential and applications, J. Funct. Anal. 244 (2007), 95-118.

[18] J. Jang, Vlasov-Maxwell-Boltzmann diffusive limit, Arch. Rational Mech. Anal. 194 (2009), 531-584.

[19] S. Kawashima, Systems of a hyperbolic-parabolic composite type, with applications to the equations of magnetohydrodynamics, thesis, Kyoto University (1983).

[20] S. Kawashima, The Boltzmann equation and thirteen moments, Japan J. Appl. Math. 7 (1990), 301-320.

[21] T.-P. Liu, T. Yang and S.-H. Yu, Energy method for the Boltzmann equation, Physica D 188 (3-4) (2004), 178-192.

[22] T.-P. Liu and S.-H. Yu, Boltzmann equation: micro-macro decompositions and positivity of shock profiles, Commun. Math. Phys. 246 (1) (2004), 133-179.

[23] C. Mouhot and L. Neumann, Quantitative perturbative study of convergence to equilibrium for collisional kinetic models in the torus, Nonlinearity 19 (4) (2006), 969-998.

[24] R.M. Strain and Y. Guo, Almost exponential decay near Maxwellian, Comm. P.D.E. 31 (2006),417-429.

[25] R.M. Strain and Y. Guo, Exponential decay for soft potentials near Maxwellian, Arch. Ration. Mech. Anal. 187 (2008), 287-339.

[26] S. Ukai, On the existence of global solutions of mixed problem for non-linear Boltzmann equation, Proceedings of the Japan Academy 50 (1974), 179-184.

[27] S. Ukai and T. Yang, The Boltzmann equation in the space $L^{2} \cap L_{\beta}^{\infty}$ : Global and timeperiodic solutions, Analysis and Applications 4 (2006), 263-310.

[28] C. Villani, Hypocoercivity, Memoirs Amer. Math. Soc., 202 (2009), iv+141. 